\title{
Comparative Chromosome Painting in Carnivora and Pholidota
}

\author{
P.L. Perelman ${ }^{a} \quad$ V.R. Beklemisheva ${ }^{a} \quad$ D.V. Yudkin ${ }^{a} \quad$ T.N. Petrina ${ }^{b} \quad$ V.V. Rozhnov ${ }^{b}$ \\ W. Nie $^{c}$ A.S. Graphodatsky ${ }^{a}$ \\ anstitute of Molecular and Cellular Biology, Siberian Division of Russian Academy of Sciences, Novosibirsk, and \\ ${ }^{\mathrm{b}}$ A.N. Severtsov Institute of Ecology and Evolution, Russian Academy of Sciences, Moscow, Russia; ' State Key \\ Laboratory of Genetic Resources and Evolution, Kunming Institute of Zoology, Chinese Academy of Sciences, \\ Kunming, PR China
}

\section{Key Words}

Ancestral Carnivora karyotype $\cdot$ Canidae $\cdot$ Feliformia

Genet - Karyotype evolution - Mustelidae - Painting probe • Pangolin genome sequencing data is discussed in the context of solving the questions that are beyond resolution of conventional banding techniques and chromosome painting.

Copyright $\odot 2012$ S. Karger AG, Basel

There are 2 major branches on the Carnivora tree Feliformia and Caniformia. Over 50 species were studied in the order Carnivora by chromosome painting (table 1). In these studies, human painting probes and painting probes from 9 carnivores (table 2) were used to define chromosome homologies between human and carnivores and among different carnivore species. Human painting probes link the painting information in Carnivora to other orders and permit hypotheses about the ancestral karyotype for the entire order. The ancestral carnivore karyotype (ACK) is highly conserved and originated from the proposed boreoeutherian karyotype by the following fusions of human chromosomes/arms: $3+19 p$, $2+20$, and $18+22$ along with other associations common to all boreoeutherians (HSA3/21, 4/8p, 7b/16p, 10p/12a/22a, 12b/22b, 14/15, 16q/19q) [Froenicke, 2005]. Comparative G-banding studies predicted, and later chromosome painting studies confirmed, 2 major modes

\section{KARGER}

Fax +4161306 1234

E-Mail karger@karger.ch

www.karger.com
(C) 2012 S. Karger AG, Basel

$1424-8581 / 12 / 1374-0174 \$ 38.00 / 0$

Accessible online at:

www.karger.com/cgr
A.S. Graphodatsky

Institute of Molecular and Cellular Biology

Siberian Division of Russian Academy of Sciences

Lavrentieva 10, RU-630090 Novosibirsk (Russia)

Tel. +7 383333 3857, E-Mail graf@mcb.nsc.ru 
Table 1. Chromosome painting in Carnivora and Pholidota

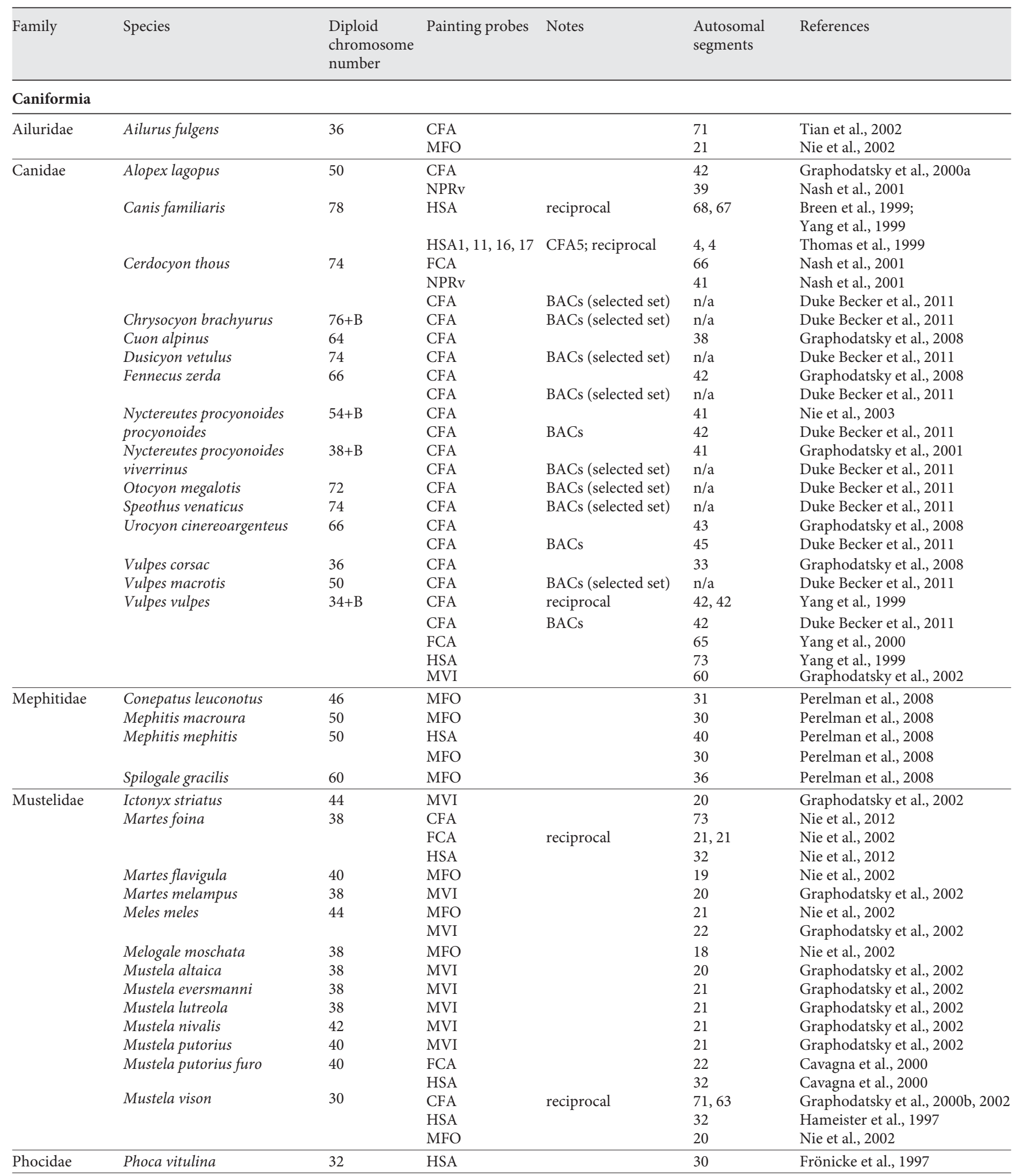


Table 1 (continued)

\begin{tabular}{|c|c|c|c|c|c|c|}
\hline Family & Species & $\begin{array}{l}\text { Diploid } \\
\text { chromosome } \\
\text { number }\end{array}$ & Painting probes & Notes & $\begin{array}{l}\text { Autosomal } \\
\text { segments }\end{array}$ & References \\
\hline \multirow[t]{6}{*}{ Procyonidae } & Bassariscus astutus & 38 & FCA & & 21 & Nash et al., 2008 \\
\hline & & & NPRv & & 61 & Nash et al., 2008 \\
\hline & Procyon lotor & 38 & CFA & & 69 & Nie et al., 2012 \\
\hline & & & HSA & & 31 & Perelman et al., 2008 \\
\hline & & & FCA & & 21 & Perelman et al., 2008 \\
\hline & & & MFO & & 18 & Perelman et al., 2008 \\
\hline \multirow[t]{8}{*}{ Ursidae } & Ailuropoda melanoleuca & 42 & CFA & & 74 & Nie et al., 2012 \\
\hline & & & FCA & reciprocal & 38,39 & Nash et al., 1998 \\
\hline & & & NPRv & reciprocal & 70,72 & Nash et al., 2001 \\
\hline & & & HSA & & 44 & Nash et al., 1998 \\
\hline & Helarctos malayanus & 74 & CFA & & 73 & Tian et al., 2004 \\
\hline & & & AME & & 38 & Nash et al., 1998 \\
\hline & Tremarctos ornatus & 52 & CFA & & 72 & Yang and Graphodatsky, 2004 \\
\hline & & & FCA & selected probes & & Nash et al., 1998 \\
\hline \multicolumn{7}{|l|}{ Feliformia } \\
\hline \multirow[t]{11}{*}{ Felidae } & Felis catus & 38 & CFA & reciprocal & 68,65 & Yang et al., 2000 \\
\hline & & & CFA & CFA5 & 4 & Thomas et al., 1999 \\
\hline & & & HSA & reciprocal & $\begin{array}{l}31 \\
(101 \mathrm{CSO})\end{array}$ & $\begin{array}{l}\text { Rettenberger et al., 1995; } \\
\text { (Wienberg et al., 1997) }\end{array}$ \\
\hline & & & MMA & reciprocal & 30,30 & Perelman et al., 2008 \\
\hline & & & MME & reciprocal & 30,30 & Perelman et al., 2008 \\
\hline & & & MVI & & 21 & Hameister et al., 1997 \\
\hline & & & & & & Graphodatsky et al., 2002 \\
\hline & & & NPRv & reciprocal & 67,65 & Nash et al., 2001 \\
\hline & $\operatorname{Lynx} \operatorname{lyn} x$ & 38 & CFA & & 69 & Nie et al., 2012 \\
\hline & Neofelis nebulosa & 38 & CFA & & 69 & Tian et al., 2004 \\
\hline & Panthera leo & 38 & CFA & & 69 & Tian et al., 2004 \\
\hline \multirow[t]{3}{*}{ Herpestidae } & Helogale parvula & 36 & FCA & & 22 & Nash et al., 2008 \\
\hline & & & NPRv & & 63 & Nash et al., 2008 \\
\hline & Herpestes javanicus & 36 & CFA & & 68 & Nie et al., 2012 \\
\hline \multirow[t]{3}{*}{ Hyaenidae } & Crocuta crocuta & 40 & CFA & & 69 & Perelman et al., 2005 \\
\hline & & & HSA & & 31 & Perelman et al., 2005 \\
\hline & & & MVI & & 23 & Perelman et al., 2005 \\
\hline \multirow[t]{2}{*}{ Eupleridae } & Fossa fossana & 42 & FCA & & 21 & Nash et al., 2008 \\
\hline & & & NPRv & & 62 & Nash et al., 2008 \\
\hline \multirow[t]{6}{*}{ Viverridae } & Paguma larvata & 44 & CFA & & 67 & Perelman et al., 2005 \\
\hline & & & HSA & & 31 & Perelman et al., 2005 \\
\hline & & & MVI & & 22 & Perelman et al., 2005 \\
\hline & Paradoxurus hermaphroditus & 42 & CFA & & 67 & Nie et al., 2012 \\
\hline & Viverricula indica & 36 & CFA & & 68 & Nie et al., 2012 \\
\hline & Genetta pardina & 52 & MFO & & 40 & this paper \\
\hline
\end{tabular}

Painting of Carnivora probes onto species from other orders

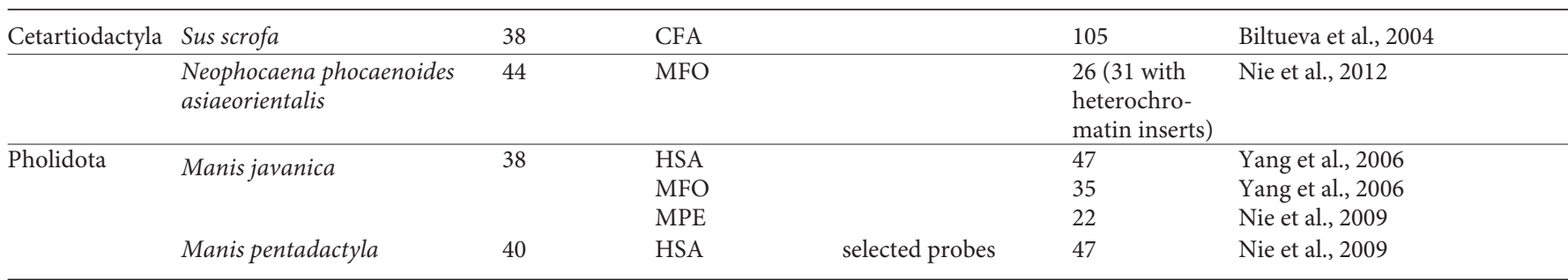

$\mathrm{AME}=$ Ailuropoda melanoleuca $; \mathrm{CFA}=$ Canis familiaris $; \mathrm{FCA}=$ Felis catus $; \mathrm{HSA}=$ Homo sapiens $; \mathrm{MFO}=$ Martes foina MMA = Mephitis macroura; $\mathrm{MME}=$ Mephitis mephitis $; \mathrm{MPE}=$ Manis pentadactyla $; \mathrm{MVI}=$ Mustela vison; $\mathrm{NPRv}=$ Nyctereutes procyonoides viverrinus. 
Table 2. Carnivora painting probe sets

\begin{tabular}{llll}
\hline Species & Abbreviation & 2n & Published in \\
\hline Carnivora & & & \\
Ailuropoda melanoleuca & AME & 42 & Nash et al., 1998 \\
Canis familiaris & CFA & 78 & Breen et al., 1999; Yang et al., 1999 \\
Felis catus & FCA & 38 & Wienberg et al., 1997; Nash et al., 1998 \\
Martes foina & MFO & 38 & Nie et al., 2002 \\
Mephitis macroura & MMA & 50 & Perelman et al., 2008 \\
Mephitis mephitis & MME & 50 & Perelman et al., 2008 \\
Mustela vison & MVI & 30 & Graphodatsky et al., 2002 \\
Nyctereutes procyonoides viverrinus & NPRv & $38+B$ & Nash et al., 2001 \\
Vulpes vulpes & VVU & $34+\mathrm{B}$ & Yang et al., 1999 \\
\hline Pholidota & & & \\
Manis pentadactyla & MPE & 40 & Nie et al., 2009 \\
\hline Primates & & & \\
Homo sapiens & HSA & 46 & Ferguson-Smith, 1997 \\
\hline
\end{tabular}

of chromosome evolution in Carnivora - slow (species that retained the ancestral carnivore karyotype with few changes) and extremely fast (genomes that were drastically reshuffled to produce chromosome complements of extant species). Feliformia in general have very conserved karyotypes that differ by few interchromosomal rearrangements. Most of the Caniformia families are also characterized by slow rates of chromosomal rearrangements with few exceptions: Canidae, Ursidae and Mephitidae whose genomes required more than 40,17 and 17 changes, respectively, from the ancestral carnivore karyotype. These are families with a so-called catastrophic rate of chromosomal rearrangements [Grafodatsky and Biltueva, 1987].

Chromosome painting data for the Feliformia and Caniformia branches were recently reviewed [Nie et al., 2012]. A detailed tree depicting chromosomal evolution of both branches of Carnivora was presented in that study. There are some interesting inversions that have been revealed by dog painting probes across the whole order [Nie et al., 2012]. In this review, we discuss the ancestral carnivore karyotype, describing some problematic ACK chromosomes, and the chromosomal evolution of Canidae as revealed by chromosome painting and BAC mapping. We report chromosome painting data on the first species among feliforms with a fast rate of chromosomal evolution (Genetta pardina). We indicate some groups within the Carnivora that might benefit from additional chromosome painting studies. In addition, chromosome evolution in a sister order of Carnivora, the Pholidota, is also reviewed.

Comparative Chromosome Painting in Carnivora and Pholidota

\section{Ancestral Carnivore Karyotype}

Decades ago extreme karyotypic conservation in Carnivora at the chromosome band level allowed identification of chromosomes 'shared' by many Carnivora species and the first attempts to identify the ancestral state of the chromosomes for the whole order [Wurster-Hill and Gray, 1975]. Later, chromosome painting in Carnivora species with conserved karyotypes helped confirm conclusions from classical cytogenetics. On the other hand, without chromosome painting it was impossible to reveal the detailed picture of complex rearrangements in groups with fast chromosome evolution such as Ursidae, Canidae, and Mephitidae. Inversions in conserved chromosomes were also hard to detect using only banding.

The ancestral karyotype of Carnivora has been described or discussed several times [Wurster-Hill and Gray, 1975; Dutrillaux and Couturier, 1983; Fronicke et al., 1997; Murphy et al., 2001b; Nash et al., 2008; Perelman et al., 2008; Nie et al., 2012]. Table 3 provides the correspondence of ACK chromosomes with feline (Felis catus, FCA), dog (Canis familiaris, CFA), stone marten (Martes foina, MFO) and human (Homo sapiens, HSA) chromosomes as well as with previously suggested ancestral carnivore karyotypes. Due to the slow rate of chromosome evolution in major Carnivora groups and the conserved pattern of G-bands across many species, even predictions based on banding techniques alone were quite precise. Chromosome painting data allowed an unequivocal deduction of the ancestral state of most chromosomes for the whole order.

Cytogenet Genome Res 2012;137:174-193 


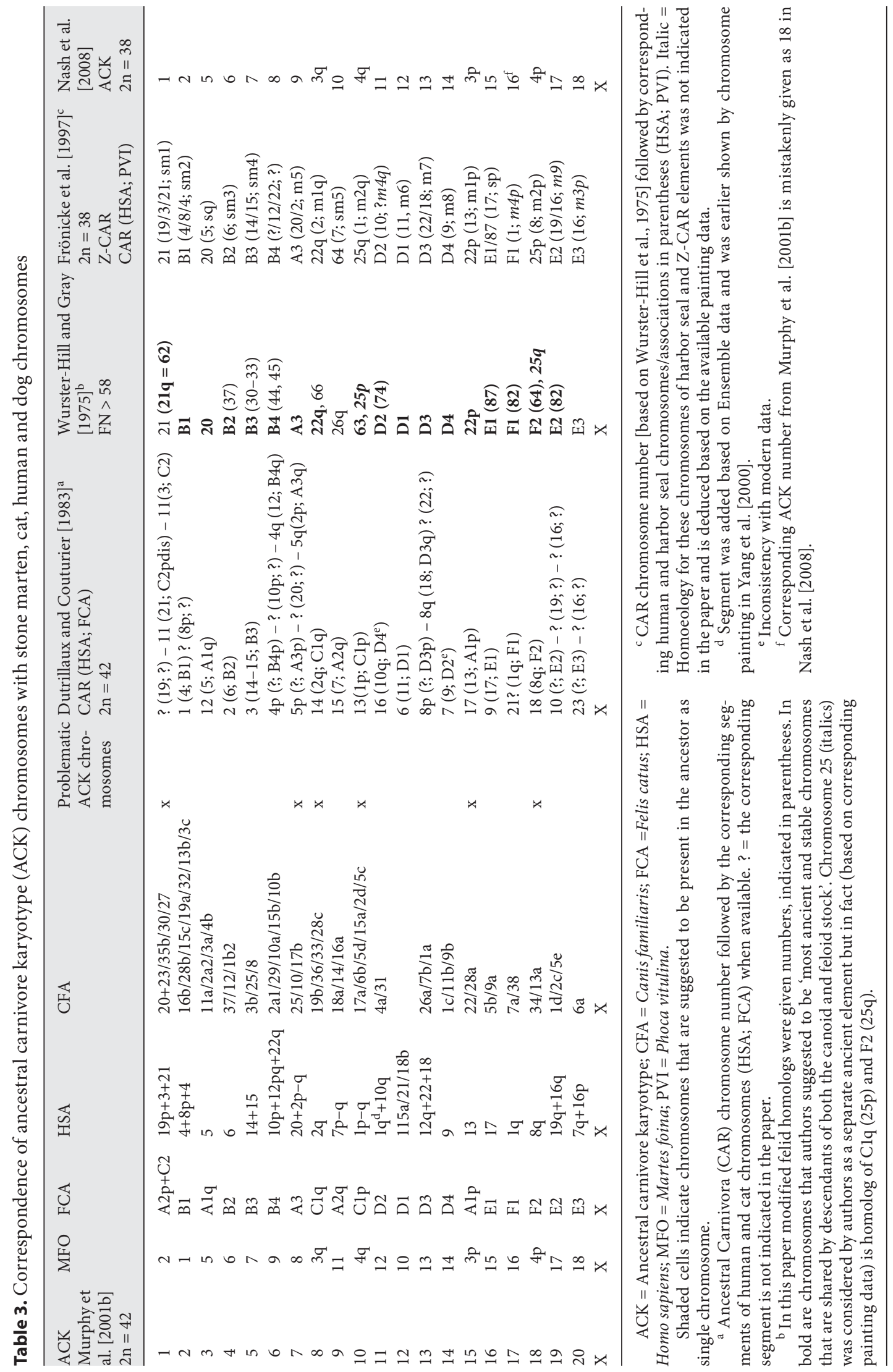




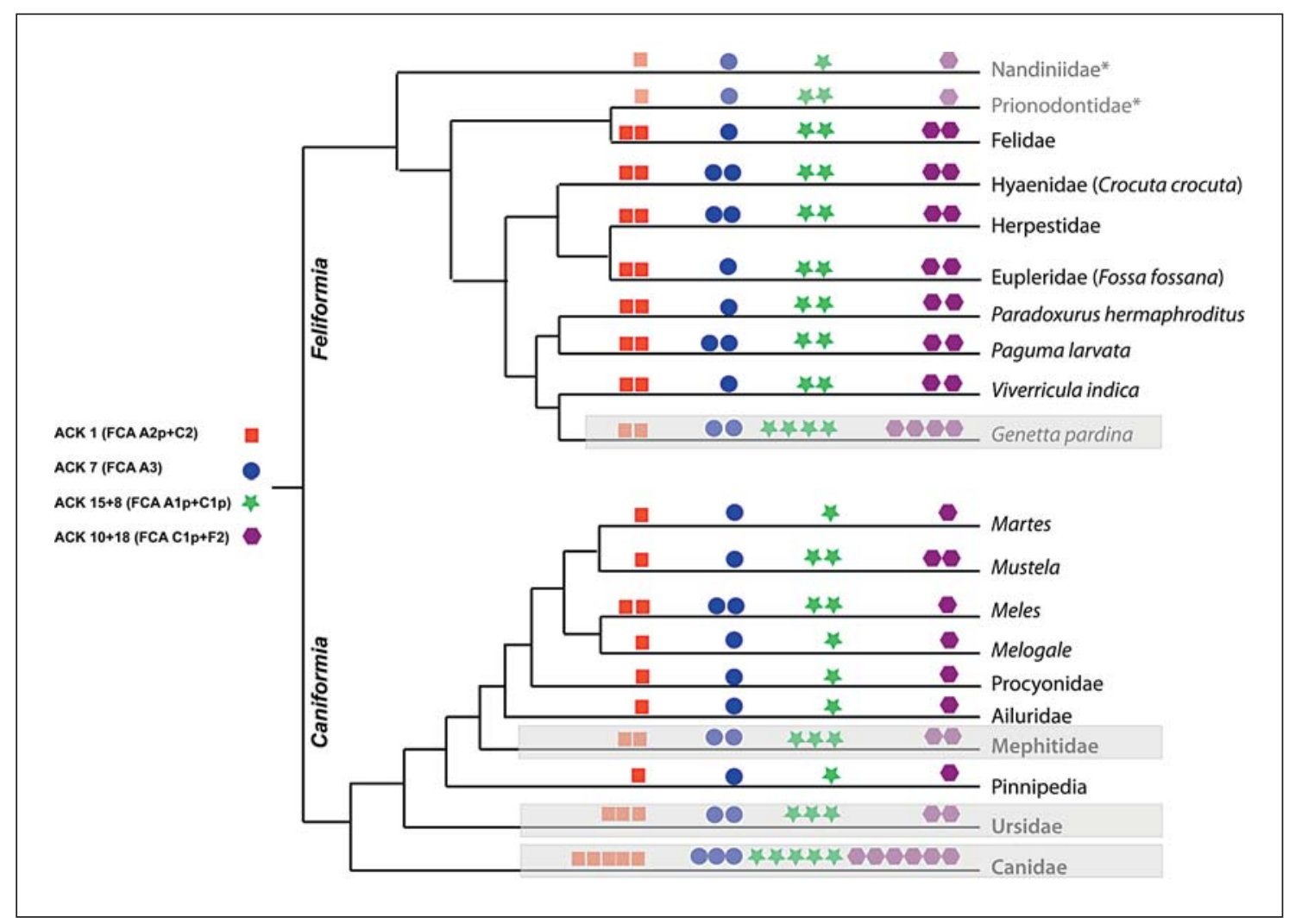

Fig. 1. The state of problematic ancestral chromosomes across Carnivora. ACK chromosome state for each group/species is based on chromosome painting data listed in table $1 .{ }^{*}$ For Nandiniidae and Prionodontidae the chromosomal state is based on G-banding data [Wurster-Hill and Gray, 1975]. Phylogenetic tree topology for Carnivora [Eizirik et al., 2010] and additionally for viverrids [Gaubert and Cordeiro-Estrela, 2006] is drawn based on modern molecular phylogenies. In grey boxes are taxa with fast rates of genome reorganization. Number of symbols on each branch indicates the number of fragments of the particular chromosome in the karyotype.
However, there are some problematic questions that remain to be resolved and which contribute to the differences in proposed diploid numbers for the ancestral carnivore karyotype, i.e. $2 \mathrm{n}=42$ and $2 \mathrm{n}=38$ (fig. 1, table 3 ). While reconstructing ancestral states one should consider phylogenetic branching of the families [Eizirik et al., 2010]. Nie et al. [2012] provided a detailed figure of karyotype relationships in Carnivora mapped onto a phylogenetic tree. In general, it is agreed that there are 2 major groups in the order, Caniformia and Feliformia. The deduction would include cladistic assumptions if the same chromosomal state occurs in both feliforms and caniforms, or if basal species of either group display the same state of the chromosome as seen in another group, meaning that this state was likely ancestral for the whole order. One has to keep in mind that there are at least 3 families in Carnivora which display severely fissioned karyotypes relative to the carnivore ancestor, and thus the absence of a particular fusion in their karyotypes has less value than its absence in the conserved karyotype. Here we use the nomenclature of Murphy et al. [2001b] for ACK chromosomes.

ACK1 (FCA A2p+C2) represents a long-standing problem in defining the ancestral carnivore karyotype, and it is also one of the signature associations of human chromosomes for the whole order Carnivora $($ HSA19p $+3 / 21)$. This ancestral chromosome is present as 2 fragments in all carnivores except for the following families on the Caniformia branch: Pinnipedia, Ailuridae, Procyonidae, Mustelidae (except Meles meles) (fig. 1). Based on G-banding comparative studies in Nandinia binotata (basal branch of Feliformia), the ACK1 chromosome is described as 1 piece. Some authors propose that this state was ancestral [Wurster-Hill and Gray, 1975; 
Fronicke et al., 1997; Nash et al., 2008]. However, the discussion about whether the fission or fusion of ACK1 represents the ancestral state will not be settled until chromosome painting is done to verify the G-banding data or until another species is identified with FCA A2p+C2 as 1 chromosome within the Feliformia branch.

ACK7 (FCA A3; HSA20+2pq prox $_{\text {) }}$ is another problematic ancestral chromosome. This case is even more complicated as it is present as 1 piece in all studied Feliformia except Herpestidae, Hyaenidae, Paguma and in all conserved caniforms (except M. meles) (fig. 1). This chromosome may be prone to breakage. It was suggested by Nie et al. [2012] that it could have been present as 2 pieces in the ancestral carnivore karyotype because it occurs as 2 pieces in outgroup species (human, pangolin, cetartiodactyls) as well as in different carnivore families. However, even though ACK7 is split in all outgroups, it is still often present as 1 chromosome on both branches of carnivores and might therefore have been present as single chromosome in the Carnivora ancestor.

ACK13 (FCA D3; HSA12 $\mathrm{q}_{\text {dist }} / 22 \mathrm{q}_{\text {prox }} / 18$ ) is present as 2 segments in the highly rearranged genomes of species within the Canidae, Ursidae and Mephitidae and in $\mathrm{Ge}$ netta; however, it is 1 single element in all other groups. The position of the centromere in ACK13 differs among species; it is at the end of the segment in American mink Neovison (Mustela) vison, in the middle of the segment corresponding to CFA26 in most of the carnivores, and it is in the middle of the segment corresponding to CFA7 in red panda and human. This example shows that even in the branches of the order with highly conserved genomes repositioning of a centromere occurs quite often. The phenomenon of neocentromere formation is absolutely worth studying in Carnivora using a whole-genome sequencing approach in combination with detailed BACmapping and sequencing [Rocchi et al., 2012].

There are also 4 ancestral elements, ACK15 (FCA Alp; HSA13) + ACK8 (FCA C1q; HSA2q) and ACK10 (C1p; HSA1pq) + ACK18 (FCA F2; HSA8q) that appear as 1 or 2 segments throughout the order (not considering groups with highly rearranged genomes). To clarify the ancestral form of these chromosomes will require additional data, especially painting of $N$. binotata chromosomes (fig. 1). ACK15+ACK8 often appears as 1 chromosome among caniforms (except Mustela and M. meles) and is uniformly present as 2 segments among studied Feliformes (except basal Nandinia). A similar situation is ACK10+ ACK18 which is present as 2 segments in all Feliformes (except Prionodon and Nandinia) and 1 segment in Caniformes (except Mustela).
Overall, for ACK1, 7, 15+8, 10+18 it is hard to determine the ancestral state based on current painting data. If the G-banding data on Nandinia is eventually confirmed by chromosome painting, then a single-segment state is more likely for all 4 chromosomes [Wurster-Hill and Gray, 1975]. Another consideration is that the recurrent fusion of the same ancestral segments is less likely than recurrent fissions. Yet another consideration in such complicated cases might be so-called hemiplasy, ancestral polymorphism persisting into modern taxa [Avise and Robinson, 2008; Robinson et al., 2008]. Until today there is no explanation for the sudden disruption of ancestral segments in canids, ursids, skunks and in genet. It will be very important to compare chromosomal breakpoints in these groups once more genomic data with chromosome assignment become available for carnivores.

By comparing a series of dog painting probes hybridized to chromosomes of different species within Carnivora, we could reconstruct the ancestral order of dog chromosomal segments on the ancestral Carnivora chromosomes (fig. 2). With additional full-genome sequences of carnivores it will be possible to discern a more detailed order of syntenic segments. With more whole-genome sequencing data appearing every day, one can expect that future comparative chromosome painting will be done computationally through genome comparison [Alekseyev and Pevzner, 2009; Kemkemer et al., 2009; Larkin et al., 2009; Ensemble Genome Browser http://www.ensembl.org/index.html]. However, among carnivores only the domestic dog (C. familiaris) genome assembly currently has full annotation linked with chromosome localizations. There is an urgent need to have additional Carnivora genomes with scaffolds assigned to chromosomes. Although publicly available, the giant panda (Ailuropoda melanoleuca) and the cat (F. catus) genomes do not have chromosome assignments that are integrated into browsers that would allow easy comparison of syntenic segments. For example, Ensemble genome browser has a very useful feature called 'comparative genomics - synteny' allowing to view in this browser 'syntenic regions that are calculated where possible from pairwise (twospecies) whole genome alignments'.

We compared results of reciprocal human-dog painting data [Yang et al., 2000] and syntenic blocks order identified in Ensemble and in Evolutionary Highway (that reflects homologous synteny blocks among $10 \mathrm{am}$ niotes (human, chimp, macaque, rat, mouse, pig, cattle, dog, opossum, and chicken) that were identified from pairwise comparisons of all genomes at $<1$ human-Mb 


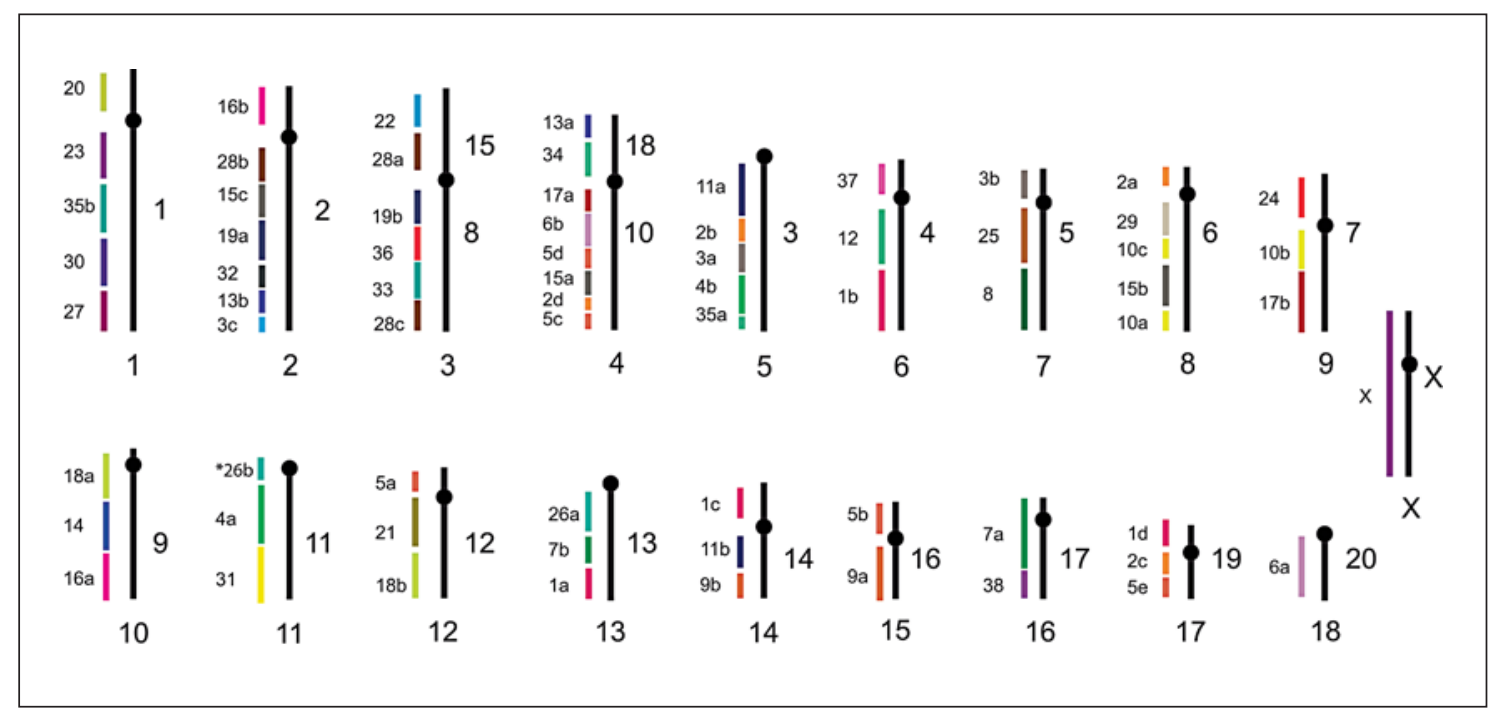

Fig. 2. Ancestral carnivore karyotype (ACK) with suggested ancestral sequence of syntenic segments corresponding to dog chromosomes based on order-wide comparison of chromosome painting data of canid painting probes. Numbers on the left indicate dog chromosomes and segments marked with letters (a, b, c, etc.) starting from the dog chromosome centromere based on recipro- cal painting data and Ensemble browser data. Numbers on the right of each ancestral chromosome are ACK chromosomes according to Murphy et al. [2001b] nomenclature. ACK chromosome numbers below correspond to Nash et al. [2008] nomenclature. The asterisk indicates a segment added based on analyses of painting and Ensemble data (see discussion). resolution (http://www.ensembl.org; http://eh-demo. ncsa.uiuc.edu/) (table 4). We aimed to identify if some large syntenic blocks were missed by chromosome painting. We disregarded segments identified in Ensemble that were $<1 \mathrm{Mb}$ in human. However, we noticed that there were many small syntenic segments around centromeric and telomeric regions which are shown on Ensemble syntenic view, but are not present in painting data. These might be false-positive blocks that are enriched in repetitive segments. Robust assemblies of repetitive regions are required to clarify whether these syntenies are real. Among larger segments, one was detected in recent painting experiments, HSA5p (CFA35; ACK1 and 3) [Nie et al., 2012] (table 4; fig. 2). Another segment, HSAlq (CFA4; ACK11), was revealed by Yang et al. [2000], but was missed in other painting studies. It forms 1 syntenic group with HSA10q (CFA26; ACK11). One segment on HSAlp (CFA17) was detected by painting and Ensemble, but is absent in Evolutionary Highway. Another segment of CFA6 (HSA2) was only shown by painting and not by other data. Several segments (HSA3p, 3q, 8p, 14q, 20q, 22q) shown in Ensemble were small in size $(<4 \mathrm{Mb})$, had centromere or telomere localization, but were not detected by painting or Evolutionary Highway. Another group of segments were not revealed by painting, but shown in
Ensemble and often in Evolutionary Highway (HSA1q, $4 q, 5 q, 7 p, 8 p, 10 q, 11 p, 15 q)$. These might represent ancestral syntenic segments that were split into small pieces on human chromosomes due to inversions. Analyses showed how precise and robust the chromosome painting tool is in describing overall chromosome evolution and paving the way to a detailed view into evolutionary reorganization of the genome.

\section{Felidae}

The family Felidae is well-known for its remarkable karyotype conservation [Wurster-Hill and Gray, 1973]. The whole family has $2 \mathrm{n}=38$, except species from the ocelot lineage that have $2 \mathrm{n}=36$ [Wurster-Hill and Centerwall, 1982; Johnson et al., 2006]. Three fusions and 1 inversion are common to 4 species studied with chromosome painting (African lion, cat, clouded leopard, Eurasian lynx) and were revealed as ACK1p+9, 3+15, 8+10, inversion ACK2 [Wienberg et al., 1997; Tian et al., 2004; Nie et al., 2012]. Earlier G-banding studies showed that chromosomes of all felids had almost identical G-banding patterns [Wurster-Hill and Centerwall, 1982]. Later chromosome painting studies with high-resolution painting probes did not show any intrachromosomal changes in the few studied felid species (table 1). How- 
Table 4. Syntenic blocks of dog (CFA) chromosomes identified by Ensemble and/or Evolutionary Highway on human (HSA) chromosomes in addition to painting data

\begin{tabular}{|c|c|c|c|c|c|c|}
\hline HSA & $\mathrm{CFA}^{\mathrm{a}}$ & $\begin{array}{l}\text { Size, Mb } \\
\text { HSA/CFA }\end{array}$ & $\begin{array}{l}\text { Localization } \\
\text { HSA/CFA }^{\text {b }}\end{array}$ & $\begin{array}{l}\text { Chromosome } \\
\text { painting HSA-CFA } \\
\text { [Yang et al., 2000] }\end{array}$ & $\begin{array}{l}\text { Evolutionary } \\
\text { Highway } \\
\text { [Larkin et al., 2009] }\end{array}$ & Comment $^{c}$ \\
\hline 1p: $114.2-120.7$ & $17: 65.1-67.0$ & $6.5 / 1.9$ & tel/tel & + & - & detected by painting \\
\hline 1q: $187.0-196.7$ & 38: $5.9-14.0$ & $9.7 / 8.1$ & int/int & - & + & inversion in human \\
\hline 1q: $223.7-227.7$ & $7: 40.6-43.4$ & $3.3 / 2.8$ & int/int & - & + & inversion in human \\
\hline 3p: 88.2-90.3 & $33: 3.0-4.7$ & $2.1 / 1.7$ & cen/cen & - & - & small size/distal localization \\
\hline $4 \mathrm{q}: 169.3-176.4$ & $25: 22.1-28.9$ & $7.1 / 6.8$ & int/int & - & + & inversion in human \\
\hline 5p: $0.1-14.7$ & 34: $3.1-15.1$ & $14.6 / 12.0$ & cen/tel & - & + & $\begin{array}{l}\text { revealed in recent painting experiments } \\
\text { [Nie et al., 2012] }\end{array}$ \\
\hline 5q: $177.5-180.7$ & $11: 3.2-6.5$ & $3.2 / 3.3$ & tel/cen & - & + & inversion in human \\
\hline $7 \mathrm{p}: 45.7-48.0$ & $16: 3.1-4.1$ & $2.3 / 1.0$ & cen/int & - & - & inversion in human \\
\hline 8p: $0.5-1.7$ & $37: 33.1-33.9$ & $1.2 / 0.8$ & tel/tel & - & - & small size/distal localization \\
\hline 10q: $51.9-59.6$ & 26: $34.5-40.6$ & $7.7 / 5.1$ & int/int & - & + & $\begin{array}{l}\text { this segment HSAlq is present as part of } \\
\text { HSA10q (CFA4/31 and CFA 26?) }\end{array}$ \\
\hline 10q: 89.2-91.0 & 26: $40.6-42.0$ & $1.8 / 1.4$ & int/tel & - & - & inversion in human \\
\hline $11 \mathrm{p}: 0.8-3.3$ & $18: 48.2-50.2$ & $2.5 / 2.0$ & int/int & - & - & inversion in human \\
\hline $14 \mathrm{q}: 20.2-22.0$ & $15: 20.2-21.6$ & $1.8 / 1.4$ & cen/int & - & - & small size/distal localization \\
\hline $15 \mathrm{q}: 22.8-32.6$ & $3: 34.5-42.2$ & $9.8 / 7.7$ & int/int & - & + & inversion in human \\
\hline $20 \mathrm{q}: 23.6-25.6$ & $23: 3.0-4.8$ & $2.0 / 1.8$ & cen/tel & - & - & small size/distal localization \\
\hline $22 \mathrm{q}: 17.5-18.7$ & $27: 47.8-48.8$ & $1.2 / 1.0$ & cen/tel & - & - & small size/distal localization \\
\hline
\end{tabular}

Only in this table we used the dog chromosome nomenclature of Breen et al. [1999].

a There is a segment of CFA6 on HSA2 according to painting [Yang et al., 2000], but it is not confirmed by Ensemble or Evolutionary Highway data.

b Segment is located near the centromere (cen), interstitially (int), or near the telomere (tel). + = Segment is present; $-=$ segment is absent.
${ }^{c}$ Inversion in human means that the corresponding dog chromosome was split into several small pieces in human (likely due to inversions) and thus was hard to reveal by chromosome painting. Small size/distal localization: those segments are present in Ensemble but not in painting or comparative mapping data. Those fragments are often just slightly bigger than $1 \mathrm{Mb}$ and are located near the centromere or telomere. We hesitate to put them on the map until further evidence is available. ever, some of the chromosomal differences between felid species were noticed in G-banding comparisons and later received confirmation by molecular methods, such as chromosome F2 in the clouded leopard [Wurster-Hill and Gray, 1973; Wurster-Hill and Centerwall, 1982; Buckley-Beason et al., 2006]. In future studies it will be very interesting to compare genomes of different felid species to reveal whether the level of conservation is also present at the gene order level. Also, it will be important to incorporate species from the ocelot lineage into new chromosome painting studies.

\section{Procyonidae}

Procyonidae is another carnivore family characterized by a uniform diploid number of $2 \mathrm{n}=38$ (at least for the species that have been karyotyped so far). Only 2 rep- resentatives of Procyonidae, both from the subfamily Procyoninae (Procyon lotor and Bassariscus astutus), have been studied by chromosome painting [Nash et al., 2008; Perelman et al., 2008]. Their karyotypes differ only by 1 inversion, revealed by dog painting probes (ACK13) [Nie et al., 2012], but otherwise they represent well the ancestral musteloid karyotype (AMK). The published banded karyotype of the basal procyonid species kinkajou (Potos flavus) appears to be identical to the karyotype of $P$. lotor, and this result is expected to be confirmed by chromosome painting [Dutrillaux and Couturier, 1983; Stanyon, 2006]. However, it will be interesting to incorporate representatives of another branch (coati (Nasua, Nasuella) + olingo (Bassaricyon)) into chromosome painting or fine mapping to see if there are cytogenetic signatures for their separate phylogenetic position, as re- 
Fig. 3. Chromosome painting of female Pardine genet (Genetta pardina, $2 \mathrm{n}=52$ ) with stone marten (Martes foina) painting probes (bars on the right of each chromosome). $\mathrm{H}$ indicates the large heterochromatin block on the heteromorphic chromosome pair.

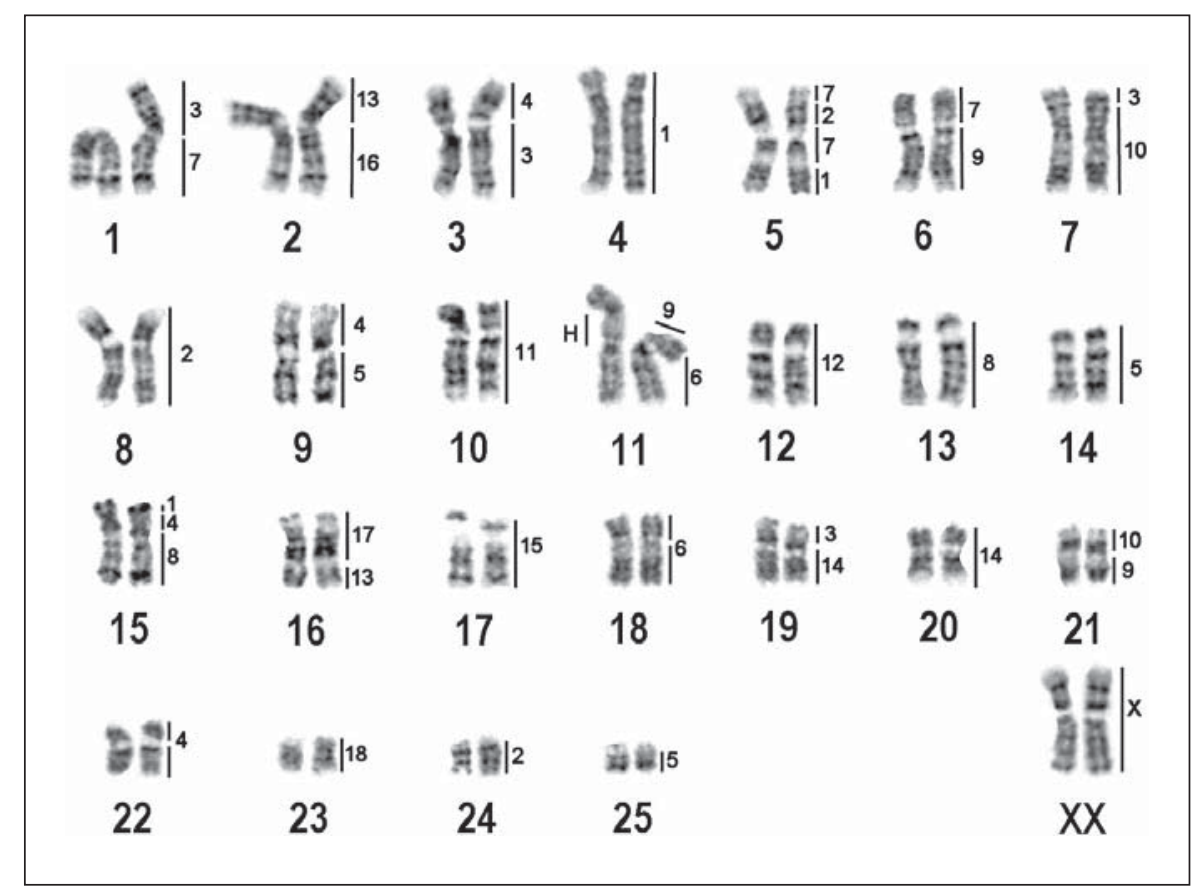

cently discovered by molecular studies [Koepfli et al., 2007]. Interestingly, according to G-banding comparison, the karyotype of Bassaricyon 'has only three felid homologs that are not considered to be modified' [Wurster-Hill and Gray, 1975].

\section{Viverridae}

The family Viverridae includes 16 genera and 36 species [Wilson and Reeder, 2005], but chromosome painting data are very scarce. So far only 4 species from Viverridae (table 1) have been studied by chromosome painting, and there appears to be no common chromosomal signature to join Viverridae with either Felidae/Prionodontidae or Hyaenidae/Herpestidae/Eupleridae. One common fusion joins Paguma larvata and Paradoxurus hermaphroditus $(\mathrm{ACK} 8+20)$ and could be signature association for the subfamily Paradoxurinae [Nie et al., 2012].

One more species from another subfamily, Viverrinae, has recently been studied by chromosome painting, $\mathrm{Vi}$ verricula indica [Nie et al., 2012]. Here we present results on another representative from Viverrinae, the Pardine genet (G. pardina, $2 \mathrm{n}=52$ ). We studied a female genet from Tchernogolovka biological station of A.N. Severtsov Institute of Ecology and Evolution, RAS, Russia, using stone marten (M. foina) chromosome painting probes described earlier [Nie et al., 2002] (fig. 3). In total, stone marten probes reveal 40 conserved autosomal segments in the genome of the Pardine genet. In Carnivora species with conserved karyotypes stone marten probes reveal 18-21 conserved segments, in skunks with rearranged karyotypes 30-36, in distant pangolin 35 conserved segments (table 1). Thus chromosome painting demonstrates that G. pardina, and possibly all members of this genus have highly rearranged karyotypes. This is the first report of feliform taxa with karyotypes not showing a high level of conservation of ACK chromosomes. Notably, Wurster-Hill and Gray [1975], based on G-banding comparative data, pointed out that Genetta tigrina has few of 'shared' Carnivora chromosomes and thus may represent yet another taxon with rearranged karyotype among otherwise conserved carnivores. Further research mapping dog painting probes will be required to precisely establish the ancestral chromosome exchange events that led to formation of the G. pardina karyotype. For example, ACK7 (FCA A3) is present as 2 segments in genet, but based on banding comparison, the breakpoint is different from other feliform species with fissioned ACK7: Crocuta crocuta, P. larvata, Helogale parvula and Herpestes javanica. We did not reveal any common rearrangement for $G$. pardina and V. indica. In this regard it would be important to study other species from the subfamily, such as those from genera Poiana and Viverra. 


\section{Other Feliformes}

Based on 2 species studied so far, there are 5 common fusions characteristic for Herpestidae (ACK11+9, 17+8, 15+7q, 20+10, 7p+1p) [Nash et al., 2008; Nie et al., 2012]. This large family requires more species to be studied by chromosome painting. For example, the genera Atilax, Bdeogale and Galidia are of special interest because comparative banding analyses revealed that their karyotypes have few 'shared' Carnivora chromosomes [Wurster-Hill and Gray, 1975]. A common inversion of ACK9 joins Eupleridae (Fossa fossana) and Herpestidae together in accordance with modern molecular phylogenies [Gaubert and Cordeiro-Estrela, 2006; Eizirik et al., 2010]. Four species of Hyaenidae have the same diploid number and are expected to have similar karyotypes based on G-banding data. The karyotype of the spotted hyena (C. crocuta) evolved from the ancestral carnivore karyotype by $4 \mathrm{fu}-$ sions (ACK8+11, 7p+10, 3+7q, 6p+20), 1 fission (ACK6) and 3 inversions (ACK1q, 12, 16) [Perelman et al., 2005; Nie et al., 2012].

Some important species that may help to define chromosomal evolution within the Feliformia branch are still lacking chromosome painting data: those are from the sister family of cats, Prionodontidae (Prionodon linsang, P. pardicolor) and the basal branch of Feliformes, Nandiniidae (N. binotata).

\section{Mephitidae}

The skunk family is one of the 3 caniform families with an increased rate of chromosomal rearrangements and includes 4 genera (Mephitis, Conepatus, Spilogale and Mydaus). Representatives of the first 3 genera have been studied by chromosome painting [Perelman et al., 2008]. Twelve fissions and 5 fusions of ancestral Carnivora chromosomes are required to form the karyotype of the skunk ancestor. A further fusion leads to Mephitis karyotypes and 5 and 2 fusions are required to form Spilogale gracilis and Conepatus leuconotus karyotypes, respectively. One common fusion was revealed for Conepatus and Spilogale $(\mathrm{ACK} 14 \mathrm{p}+4)$; however, it contradicts the association of Mephitis/Spilogale indicated by molecular phylogeny and requires additional research as the small insertion of ACK14 (FCA D4) on ACK4 may represent repetitive sequences, not revealed by C-banding [Perelman et al., 2008; Eizirik et al., 2010]. Stink badger (genus Mydaus) as basal branch of Mephitidae holds special interest for chromosome painting studies. Also it would be interesting to study South American representatives of the genus Conepatus (C. chinga, C. humboldtii, C. semistriatus).

\section{Ursidae}

Karyotypes of Ursidae were also formed by great fissioning of ancestral elements, namely 16 fissions and 1 inversion (ACK19) [Nie et al., 2012]. Of 8 extant ursid species 2 karyotypes stand out: giant panda (A. melanoleuca, $2 \mathrm{n}=42$ ) and spectacled bear (Tremarctos ornatus, $2 \mathrm{n}=$ $52)$, the rest have a high-chromosome, uniform diploid number of $2 \mathrm{n}=74$. The giant panda karyotype is formed by further 16 fusions and 1 inversion (ACK3). Ursinae and Tremarctinae are joined by 2 fissions $(\mathrm{ACK} 3,6)$ and 2 fusions (ACK17+20, 1p+16p) and this forms karyotypes of ursins [Nash et al., 1998, 2001; Tian et al., 2004; Yang and Graphodatsky, 2004]. The karyotype of spectacled bear is further formed by 1 inversion and 11 more fusions that are different from those found in giant panda [Yang and Graphodatsky, 2004].

\section{Musteloidea}

Mustelidae is one of the families that are best studied by chromosome painting among carnivores (13 species; table 1). It was already known for its karyotype conservation well before chromosome painting was established [Wurster-Hill and Centerwall, 1982; Graphodatsky et al., 1989]. Detailed G-banding comparative analyses even allowed deduction of the ancestral musteloid karyotype; it was suggested that it could be Martes-like [Graphodatsky et al., 1989], which was later confirmed by chromosome painting [Graphodatsky et al., 2002]. Only recent chromosome painting studies with dog paints showed that seemingly conserved musteloid karyotypes do have differences, i.e. inversions. For example, the $M$. foina karyotype has all the same syntenic groups as the ancestral carnivore karyotype, but differs by 6 inversions revealed by dog painting probes [Nie et al., 2012]. Large heterochromatic blocks, present as whole chromosome arms, contribute to further differences between conserved musteloid karyotypes [Graphodatsky et al., 2002]. In a recent molecular phylogeny of Mustelidae, Koepfli et al. [2008] provided support for 8 subfamilies. Lutrinae is one of the subfamilies with no painting data and only comparative G-banding data available and remarkable uniformity of the chromosome complement: all studied species have $2 \mathrm{n}=38$. The karyotype of Lutra lutra is very similar to the ancestral musteloid karyotype based on Gbanding analyses. However, it would be interesting to study Lutrinae with dog paint probes.

In Mustelinae, the American mink (M. vision) has a basal position and, unusual for Mustelidae, a low diploid chromosome number of $2 n=30$ caused by 6 fusions of ancestral chromosomes, accompanied by 3 inversions
Perelman/Beklemisheva/Yudkin/ Petrina/Rozhnov/Nie/Graphodatsky 
(ACK3, 2, 6). Fission of ACK2 is characteristic for the rest of Mustela species and further evolution involves $3 \mathrm{fu}-$ sions (ACK8+18, 2q+12, 9+11) for M. eversmanni and $M$. putorius (reverse fission of ACK2q-12) and 1 fusion $($ ACK11+18) leading to M. altaica. In Galictinae, zorilla (Ictonyx striatus, painting data) and marbled polecat (Vormela peregusna, banding data) have, except heterochromatin additions that are particularly large in zorilla, an unchanged ancestral musteloid karyotype [Graphodatsky et al., 2002]. It would be interesting to incorporate karyotypes of the third genus of this subfamily (neotropical Galictis) into chromosome painting, as it was predicted by G-banding in $G$. vittata that it has a unique karyotype despite the same diploid number of $2 n=38$ [Wurster-Hill and Centerwall, 1982].

In Helictidinae, Melogale moschata also has retained the ancestral musteloid karyotype [Nie et al., 2002]. In Martinae, all Martes species with $2 \mathrm{n}=38$ are likely to have retained the ancestral musteloid karyotype, as has been confirmed for $M$. foina (with 6 inversions, painting), M. melampus (painting), M. zibellina, and M. martes (banding). An additional further fission of ACK5 is characteristic for karyotypes of M. flavigula $(2 \mathrm{n}=40)$ and Gulo gulo ( $2 \mathrm{n}=42$, and also fission of ACK13) [Graphodatsky et al., 2002; Nie et al., 2002]. The tyara (Eira barbara, $2 \mathrm{n}=38$ ) chromosomes were also studied by Gbanding and were shown to have some differences with other mustelids (inversions?) [Wurster-Hill and Centerwall, 1982]. The true Melinae species M. meles $(2 \mathrm{n}=44)$ has a karyotype that is formed by fission of 3 'problematic' ancestral chromosomes (ACK1, 7, 8+15) [Graphodatsky et al., 2002]. It would be interesting to know whether these 3 fissions are characteristic for the whole subfamily by studying the hog badger (Arctonyx collaris) karyotype.

Basal species of Mustelidae, the American badger (Taxidea taxus, $2 \mathrm{n}=32$ ) and honey badger (Mellivora capensis, $2 \mathrm{n}=40$ ), were shown to have unique karyotypes based on G-banding and are particularly interesting for future chromosome painting studies [Wurster-Hill, 1973; Wurster-Hill and Centerwall, 1982].

The karyotype of the red panda (Ailurus fulgens, $2 \mathrm{n}=$ 36 , Ailuridae) differs just slightly from the ancestral carnivore karyotype, by 1 fusion (ACK5+20) and 4 inversions revealed by dog painting probes (ACK1, 2, 6, 8+15) [Nie et al., 2002; Tian et al., 2002]. Pinnipedia karyotypes are well-known for their remarkable chromosomal conservation, described by Arnason [1977]. However, just 1 species has been studied by chromosome painting, Phoca vitulina $(2 \mathrm{n}=32)$ [Fronicke et al., 1997]. Three fusions of ancestral chromosomes $(\mathrm{ACK} 3+16,6+20,11+17)$ form its karyotype. The other 2 families, Otariidae and Odobenidae, have not been studied by chromosome painting. It would be interesting to check if there are any inversions in pinniped karyotypes; these could be revealed by hybridizing dog painting probes.

\section{Chromosome Evolution in Canidae}

The Canidae family comprises 13 genera and 35 species and has been extensively studied by chromosome painting [Wilson and Reeder, 2005]. Domestic dog, C. familiaris, a member of the family, has been notably difficult to characterize on the chromosomal level because of its karyotype, consisting of 38 acrocentric autosomes. There were early attempts to establish an international standard karyotype for dog, but it was virtually impossible to reliably identify all chromosomes based on the banding alone, especially for the smallest acrocentric chromosomes [Breen, 2008]. Only in 1999, when a combination of painting probes from both $\operatorname{dog}(2 \mathrm{n}=78)$ and fox $(2 \mathrm{n}=34+\mathrm{B}$, easily indefinable chromosomes) was used in cross-species chromosome painting studies (human-dog-fox), the puzzle of dog chromosome identification was solved. Then this nomenclature was linked to radiation-hybrid $(\mathrm{RH})$ mapping and linkage mapping data of the domestic dog [Yang et al., 1999; Sargan et al., 2000]. At the same time, independent reciprocal dog-human painting, later combined with cosmid mapping, also produced molecularly-linked dog chromosome nomenclature, which is currently used in the genomic assembly of the domestic dog [Breen et al., 2001; Lindblad-Toh et al., 2005]. The conversion tables between 2 painting-produced dog chromosomal nomenclatures have been previously described [Graphodatsky et al., 2000a; Breen, 2008]. In this paper we use the nomenclature by Yang et al. [1999].

To date, dog chromosome painting probes have been applied to the chromosomes of 10 canid species, uncovering an incredible picture of chromosomal reshuffling during the evolution of this family (table 1, fig. 4). Although we have learned a great deal from these studies, the story is still far from being complete. Extant canid speciation occurred in the last 10 million years [LindbladToh et al., 2005]. Such recent speciation has created additional difficulties for constructing phylogenies, regardless of the characters being used (sequence, morphology, cytogenetics), and was accompanied by numerous homoplasies. Therefore, phylogenetically there is still no consensus 
Fig. 4. The state of chromosomal studies in Canidae. The branching of the phylogenetic tree is shown according to LindbladToh et al. [2005] with 2 changes for more parsimonious arrangement based on cytogenetic data ( $V$. corsac is sister branch with A. lagopus/V. velox and Otocyon megalotis is sister branch to Nyctereutes, rather than separate). Diploid chromosome numbers are from http://www.bionet.nsc.ru/labs/ chromosomes/ and Sillero-Zubiri et al. [2004]. Dog chromosome nomenclature follows Yang et al. [1999]. Correspondence with Breen et al. [1999] nomenclature is provided in Breen [2008] and Graphodatsky et al. [2000a]. Recurrent rearrangement is shown in blue. In grey are species with no painting data. Black lines: species was studied by chromosome painting; dotted lines: species was studied by BAC mapping only. FU: fusion of ancestral elements, FI: fission of ancestral elements, BP: breakpoint, Inv: inversion. Arrowheads under chromosome numbers indicate direction of the ancestral segment based on BAC mapping data [Duke Becker et al., 2011]. Painting data are from papers listed in table 1.

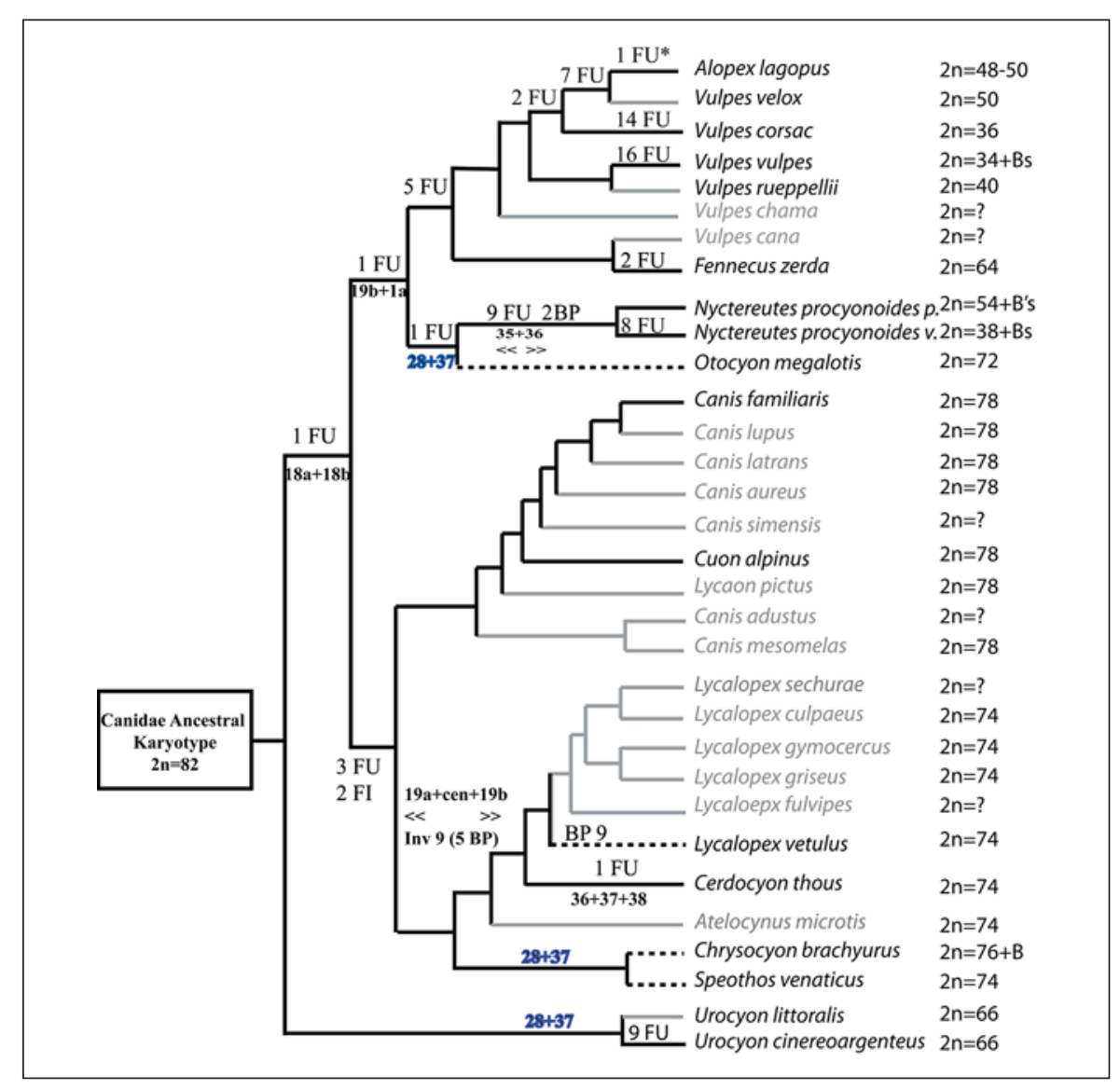

on the branching order of species within the canid family. In some aspects all phylogenies agree: there are 3 main groupings: dog-like canids (Canis, Cuon, Lycaon), red foxlike canids (Vulpes, Fennecus, Alopex), South American canids (Pseudoalopex, Lycalopex, Atelocynus, Cerdocyon, Chrysocyon, Speothos), with the most basal branch of North American foxes (Urocyon) [Zrzavy and Ricankova, 2004; Bardeleben et al., 2005; Lindblad-Toh et al., 2005]. There is still a great level of uncertainty about the exact placement of Nyctereutes, Otocyon, Speothos, Chrysocyon, branching within the Holoarctic fox clade, and branching of Canis-Cuon-Lycaon. It is possible that fine mapping across the whole family might provide cytogenetic evidence for species grouping, although it is unlikely to be helpful in case of karyotypically uniform dog-like canids, which will require molecular phylogenies with better species sampling and based on fast evolving sequences.

Chromosome painting has revealed interesting patterns of chromosome rearrangements in several branches. All dog-like canids (Canis, Cuon, Lycaon) possess karyotypes with $2 n=78$, widely identical to that of the domestic dog. Painting of the full set of dog probes onto chromosomes of Cuon alpinus confirmed that both species have indeed the same chromosomal set without interchromosomal rearrangements [Graphodatsky et al., 2008]. It is highly likely that the rest of the dog-like species with $2 n=78$ shares exactly the same set of chromosomes, as expected from G-banding comparative data [Wurster-Hill and Centerwall, 1982; Wayne et al., 1987].

In the red fox-like canids chromosome painting shows that ancestral canid elements were fused into totally different combinations in 3 of the 4 studied species. Arctic fox (Alopex lagopus) has 7 unique fusions, corsac (Vulpes corsac) has 14, and red fox has 16 fusions. Kit fox (Vulpes macrotis) and Arctic fox appear to have identical karyotypes based on G-banding comparisons [Wayne et al., 1987].

Recently, canid chromosome evolution has been studied at a higher level of resolution. Dog BACs with $10-\mathrm{Mb}$ intervals were localized on chromosomes of 3 different canid species (Nyctereutes procyonoides viverrinus, Urocyon cinereoargenteus, Vulpes vulpes). Selected sets of BACs from chromosomes with common canid break- 
Table 5. Grey fox (Urocyon cinereoargenteus, UCI) chromosome nomenclature

\begin{tabular}{llllll}
\hline $\begin{array}{l}\text { Graphodatsky } \\
\text { et al. [2008] }\end{array}$ & $\begin{array}{l}\text { Duke Becker } \\
\text { et al. [2011] }\end{array}$ & $\begin{array}{l}\text { Graphodatsky } \\
\text { et al. [2008] }\end{array}$ & $\begin{array}{l}\text { Duke Becker } \\
\text { et al. [2011] }\end{array}$ & $\begin{array}{l}\text { Graphodatsky } \\
\text { et al. [2008] }\end{array}$ & $\begin{array}{l}\text { Duke Becker } \\
\text { et al. [2011] }\end{array}$ \\
\hline $\begin{array}{l}\text { 1prox } \\
1 \text { dist }\end{array}$ & 12 & 11 & 13 & 22 & 24 \\
2 & 15 dist & 12 & 17 & 23 & 19 \\
3 & 8 & 13 & 23 & 24 & 18 \\
4 & 3 & 14 & 9 & 25 & 16 \\
5 & 7 & 15 & 11 & 26 & 29 \\
6 & 1 & 16 & 22 & 27 & 30 \\
7 & 2 & 17 & 10 & 28 & 32 \\
8 & 4 & 18 & 31 & 29 & 25 \\
9 & 5 & 19 & 20 & 30 & 15 prox \\
10 & 6 & 20 & 21 & 31 & 28 \\
\hline
\end{tabular}

points were applied to 8 more species [Duke Becker et al., 2011]. A conversion table for grey fox nomenclature [Graphodatsky et al., 2008] is shown in table 5.

BAC mapping also revealed breakpoints that mark intrachromosomal rearrangements and may serve as cytogenetic signatures (fig. 4). The formation of the Cerdocyon thous karyotype is marked by the fusion of $3 \mathrm{dog}$ chromosomes CFA36 $+37+38$ [Nash et al., 2001]. Additionally, an inversion in CFA9 and possibly other rearrangements (because according to BAC-mapping it was split into 3 chromosomes) are characteristic for this karyotype. Different breakpoints of CFA9 homologs are present in Lycalopex vetulus and yet another one in both raccoon dogs (Nyctereutes procyonoides procyonoides and viverrinus). Several breakpoints have been identified in U. cinereoargenteus. Urocyon is found at the basal branch on the canid tree. Until further evidence is made available, the most parsimonious way is to assume that grey fox rearrangements depicted on the tree are specific for this species and not for the canid ancestor. Selected BACs were mapped onto 3 South American species in which no chromosome painting studies had been performed: Chrysocyon brachyurus, L. vetulus and Speothos venaticus [Duke Becker et al., 2011]. The following rearrangements, CFA32-19a, 34-13a, 19a+19b, 13a+13b, 1a+1b, are indeed common for dog-like canids and South American canids, cytogenetically confirming this grouping.

\section{Recurrent Fusions of Ancestral Chromosomes in Canidae}

Two fusions of ancestral elements appear to be homoplasic and have not been resolved by chromosome painting. Fusion of CFA18a+38 occurred both in distantly re- lated red fox and Urocyon branches. However, BAC mapping data show that these fusions are likely independent events because 18a and 38 are fused in different directions in these species (fig. 4). This is also true for the complex ancestral chromosome composed of CFA35+36 which is present in V. vulpes and raccoon dogs. Corresponding dog chromosomes are fused by centromeres in fox and by telomeres in raccoon dogs and could have occurred independently.

In 2 other instances of assumed homoplasic fusions $(C F A 18 a / 38 / 18 b+36$ and $19 a / 32+8)$ that occurred both in arctic fox (A. lagopus) and corsac (V. corsac), we believe that the likely arrangement for the phylogeny is the sister branching of corsac with arctic fox rather than with red fox, as it is currently suggested by phylogenetic studies. This requires revision of the phylogenetic relationships of these species with more samples and molecular markers [Lindblad-Toh et al., 2005].

Duke Becker et al. [2011] also revealed one more instance of a recurrent rearrangement, CFA31+33, that occurs in the 3 branches Chrysocyon/Speothos, Nyctereutes/ Otocyon and in Urocyon. These authors speculate that it is possible that this fusion was ancestral and was lost in more derived species. It will be very interesting to determine if ancestral segments in this fusion have the same orientation.

\section{Canidae Ancestral Karyotype}

We attempted to refine the structure of the Canidae ancestral karyotype (CAK) $(2 n=82)$ by deducing the centromeric position of the ancestral elements and the orientation of corresponding dog chromosome segments based on the most recent BAC-mapping data of 3 canid 


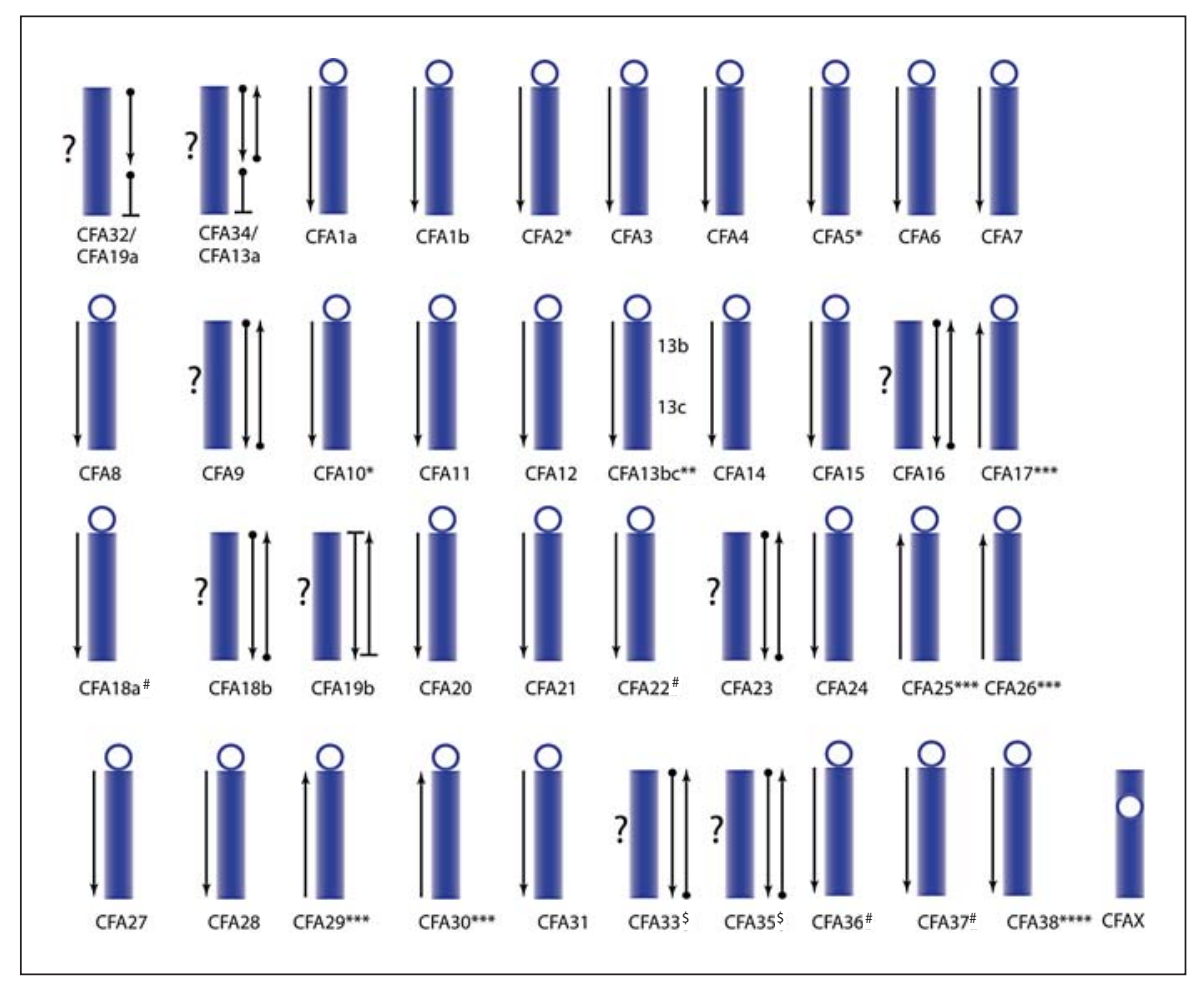

Fig. 5. Canidae ancestral karyotype (CAK). The orientation of the ancestral elements relative to the centromere in CAK is shown. Empty circles indicate deduced ancestral centromeres, small filled circles centromeres in extant species. Arrows on the left indicate direction of corresponding dog chromosomes. Arrows on the right indicate direction of the ancestral segment in extant species relative to dog. The horizontal bars indicate that this fragment in extant species is fused with another one and not in the centromere. Question mark: it is hard to deduce ancestral direction of the segment based on the currently available data. Actual chromosome size is not reflected in the figure. Dog chromosome nomenclature follows Yang et al. [1999]. * These chromosomes have breakpoints and have undergone some inversions in $U$. cinereoargenteus. ${ }^{* *}$ In Otocyon megalotis and N. pr. procyonoides the centromere is repositioned on this chromosome relative to the deduced ancestral state. ${ }^{* *}$ In dog the centromere is repositioned to another end of the ancestral segment. ${ }^{* * *}$ In N. pr. procyonoides the centromere is repositioned on this chromosome relative to the deduced ancestral state. \# In the branches of true foxes and raccoon dogs the centromere is repositioned to another end of the ancestral segment. ${ }^{\$}$ In the branch of $U$. cinereoargenteus the centromere is repositioned to another end of the ancestral segment. species (U. cinereoargenteus, $N$. procyonoides, $V$. vulpes) [Graphodatsky et al., 2008; Duke Becker et al., 2011] (fig. 5). In our Canidae ancestral karyotype, 22 ancestral chromosomes would look exactly like extant dog chromosomes. In 5 instances the centromeres on the ancestral chromosomes would be on the opposite end relative to dog. In 8 instances there is not sufficient data to deduce the ancestral position of the centromeres. The remaining chromosomes represent segments of extant dog chromosomes. Our data provides strong evidence that centromere repositioning, recently described in detail in primates, occurred quite often during canid chromosome evolution [Rocchi et al., 2012]. Striking examples of how centromeres can change their position so many times in just 10 million years of evolution are shown in figure 6 .
We anticipate that as additional canid genomes are sequenced and anchored to chromosome locations, such information will allow for the detailed reconstruction of the Canidae ancestral karyotype with the orientation of ancestral segments further identified.

B chromosomes, supernumerary highly polymorphic elements of the chromosome complement, occur among Carnivora only in canids [Switonski et al., 2009]. The composition, role and origin of $\mathrm{B}$ chromosomes are slowly getting uncovered [Camacho et al., 2000; Vujosevic and Blagojevic, 2004; Szczerbal et al., 2005]. Chromosome painting using microdissected probes of $\mathrm{B}$ chromosomes has shed light on their basic structure [Trifonov et al., 2002]. Moreover, recently it was discovered that $c K I T$ oncogene sequences are localized on the B chromosomes 


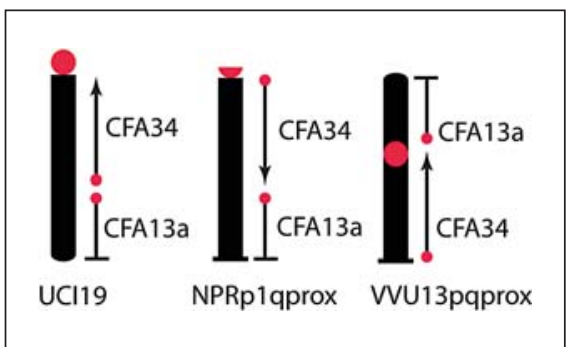

Fig. 6. Centromere repositioning in Canidae. Segment orientation is based on BAC mapping data of Duke Becker et al. [2011]. Full circle: Centromere of acrocentric chromosome; half-circle: chromosome has another arm; horizontal bar: syntenic segment does not end at telomere. VVU: Vulpes vulpes; UCI: Urocyon cinereoargenteus; NPRp: Nyctereutes procyonoides procyonoides. Dog chromosome nomenclature follows Yang et al. [1999], UCI nomenclature follows Graphodatsky et al. [2008].

of red fox and raccoon dogs [Graphodatsky et al., 2005; Yudkin et al., 2007], providing new insights into the role of B chromosomes in genome function and evolution. Furthermore, 25 BAC clones (some carrying known genes cKIT, MDN1, RET, LRIG1) have been described with duplicated localizations on both B chromosomes and autosomes in canids (N. procyonoides, V. vulpes) [Duke Becker et al., 2011]. Interesting observations were made about so-called CFA-vacant regions on the chromosomes of different species not covered by any BACs, but also were too large to be represented by centromeric sequences [Duke Becker et al., 2011].

Most of the canid species with contrasting diploid numbers are now studied either by chromosome painting or by BAC mapping. But several species still remain unstudied cytogenetically and may hold surprising rearrangements: fox-like canids Vulpes rueppellii, V. chama and $V$. cana. BAC mapping may also reveal interesting intrachromosomal rearrangements in species from other genera with the same diploid number and seemingly identical karyotypes: Canis, Lycaon, Lycalopex, Atelocynus, and Urocyon.

\section{Use of Carnivora Painting Probes to Reveal Chromosome Evolution in Other Orders}

Carnivora painting probes were also used in several studies to reveal genome-wide chromosome rearrangements in other mammalian orders: dog probes in pig (Sus scrofa, Cetartiodactyla), stone marten probes in Javan pangolin (Manis javanica, Pholidota) and in Yangtze finless porpoise (Neophocaena phocaenoides asiaeorientalis, Cetartiodactyla) [Biltueva et al., 2004; Yang et al., 2006; Nie et al., 2012]. These studies showed how informative cross-order painting analyses can be. However, it was made evident that with an increase of phylogenetic distance between species, getting strong hybridization signals became increasingly difficult. It also became apparent that getting strong hybridization signals was easier using chromosome paints derived from a species with conserved karyotype (e.g. the stone marten), than from species with a highly rearranged karyotype like the dog.

\section{Pholidota}

Pholidota is a remarkable group of mammals with striking adaptations, but it remains largely unstudied from the genetic point of view. Recently it was shown by molecular phylogenies to be sister group of Carnivora [Murphy et al., 2001a]. As yet there is no comprehensive molecular phylogeny described for this group; however, there are morphological phylogenetic studies [Gaudin et al., 2009] (fig. 7). There are a total of 8 taxonomically described species of extant pangolins: 4 of African origin (Manis tricuspis, M. tetradactyla, M. gigantea, M. temminckii) and 4 from Asia (M. javanica, M. culionensis, $M$. pentadactyla, M. crassicaudata) [Gaubert and Antunes, 2005; Wilson and Reeder, 2005; Gaudin et al., 2009]. The genome of $M$. pentadactyla is being sequenced within the frame of 29 mammalian genome projects (http://genome. wustl.edu/genomes/view/manis_pentadactyla). Complete mitochondrial genomes have been published for 2 pangolin species [Arnason et al., 2008; Qin et al., 2012]. Also, there is a valuable resource of characterized BAC library of $M$. pentadactyla with numerous clones having been mapped onto chromosomes by FISH [Che et al., 2008].

To the best of our knowledge, the diploid chromosome number for only 3 species of pangolins has been reported: $M$. javanica $(2 \mathrm{n}=38)$, M. pentadactyla $(2 \mathrm{n}=40$, with possible polymorphism $2 \mathrm{n}=38-40)$ and $M$. crassicaudata $(2 \mathrm{n}=36)$ [detailed review and references in Nie et al., 2009]. Chromosomes of M. pentadactyla also display polymorphisms in amounts of heterochromatin and differences in locations of nucleolar organizing regions. Interestingly, one of the nucleolar organizer regions in $M$. javanica is located on the Y chromosome [Nie et al., 2009].

Chromosome complements of $M$. pentadactyla and $M$. javanica differ by 3 centric fissions and 4 centric fusions (with 1 centromere repositioning event) based on chromosome painting using $M$. pentadactyla painting probes 
[Nie et al., 2009]. A full set of human painting probes was applied to chromosomes of $M$. javanica and selected human probes to chromosomes of $M$. pentadactyla [Yang et al., 2006; Nie et al., 2009]. Seven associations of human chromosomes common for the 2 species of pangolin studied by chromosome painting and potentially representing Pholidota cytogenetic signatures are: HSA2q/10q, HSAlq/11, 2p/5, 4p+q/20, 5/13, 6/19p, and 8q/10p [Nie et al., 2009].

Genomes of pangolins contain common mammalian associations: HSA3/21, 4/8, 7/16, 12/22, 14/15 and 16/19. However, chromosome painting revealed 16 other associations of human chromosomes, none of which were common for both pangolins and carnivores or for that matter for pangolins and any other mammalian order. Up to now, only Asian pangolins have been studied by chromosome painting. According to morphological data, the Asian species are more recently derived and likely diverged later than African pangolins [Gaudin and Wible, 1999]. Further studies of more basal pangolin species could potentially reveal ancestral signatures common for pangolins and carnivores.

\section{Other Resources to Complement Chromosome Painting Data}

The domestic dog genome is an excellent example of how developing essential genetic tools can advance studies in multiple species. Chromosome painting data has provided a valuable link in assignment between human and dog chromosomes, and allowed unambiguous identification of individual chromosomes in the complex karyotype of the dog [Breen et al., 1999; Yang et al., 1999]. These results have fueled numerous studies for development of more advanced molecular cytogenetics tools for identification of dog chromosomes coupled with the development of rich BAC libraries, dense RH maps, detailed FISH maps, and, most importantly, with high-coverage genomic data [reviewed in Breen, 2008]. This combination of techniques allowed the improvement of the domestic dog genome assembly, and this high-quality genomic resource allowed further expansion to benefit and accomplish comparative studies with other canids [Lindblad-Toh et al., 2005].

With the cost of whole-genome de novo sequencing rapidly coming down, one can expect more Carnivora genomes to be sequenced in the near future. Some of them are already scheduled to be sequenced in frame of an international project to sequence the genomes of

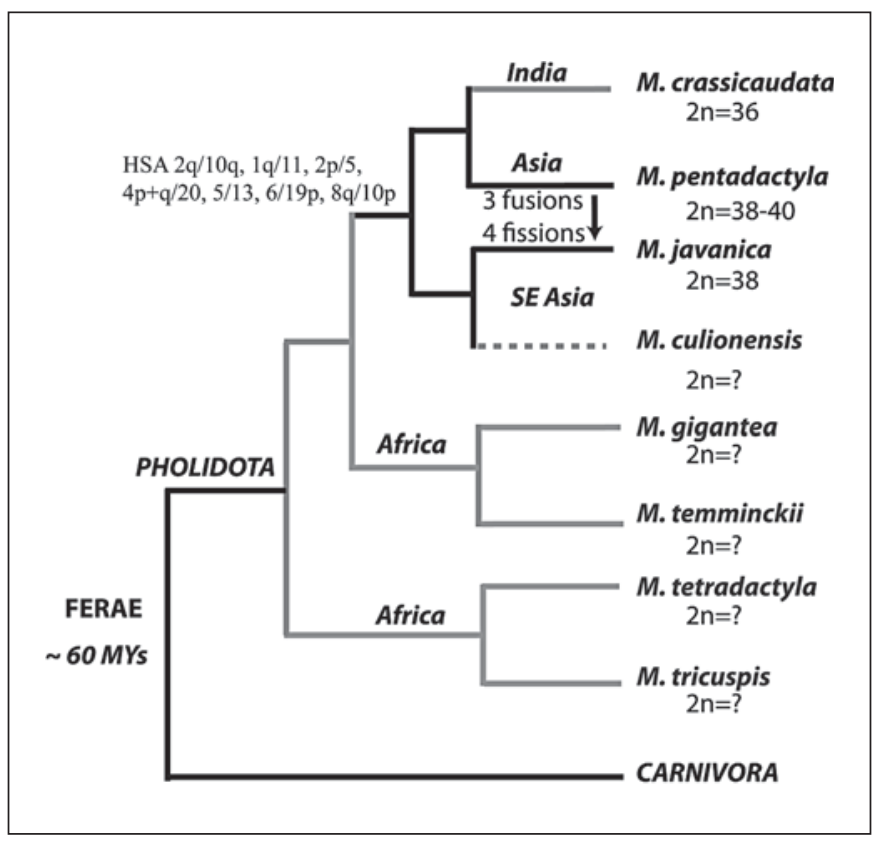

Fig. 7. The state of cytogenetic studies in Pholidota. The species arrangement on the tree is from morphology-based phylogenetic studies of Gaudin et al. [2009] and Gaudin and Wible [1999], modified with the dashed line for Manis culionensis as closely related to M. javanica [Gaubert and Antunes, 2005]. Date of divergence of Pholidota and Carnivora is from calibrated mitogenomic phylogeny [Arnason et al., 2008]. The species distribution and names are from Wilson and Reeder [2005]. Diploid chromosome number references are provided in detail in Nie et al. [2009]. Chromosome rearrangement information is based on chromosome painting data [Yang et al., 2006; Nie et al., 2009]. Grey lines represent species not studied by chromosome painting.

10,000 vertebrate species, Genome10K (http://www.genome10k.org/). For example red fox $V$. vulpes is on the list to be sequenced shortly. Full-genome sequence of the red fox will allow detailed comparison of the dog and fox genomes and will shed light onto the molecular structure of evolutionary breakpoint regions. Among upcoming or already sequenced genomes that will provide rich ground for comparison are felids (tiger, cheetah, lion, lynx, snow leopard), bears (polar bear, giant panda) and several other Carnivora species.

Two poorly studied types of chromosome evolution also occurred in Carnivora. These involve B chromosomes and long stretches of heterochromatic blocks in some chromosomes. Heterochromatin blocks often appear as whole chromosomal arms in many carnivore species (e.g. striped polecat, skunks, mountain weasel, steppe polecat, arctic fox, etc.) [Graphodatsky and Radjabli, 
1988]. Future whole-genome sequencing efforts may reveal sequences of those kinds of chromosomal elements that are clearly composed mostly of repeats. Unveiling repeat structures and sequences may identify other unique sequences that are currently overshadowed by repeats, providing more information about the origin and function of B chromosomes and heterochromatic blocks.

Another highly anticipated result from the full-genome sequencing of the red fox is providing insight into the sequence composition of B chromosomes, supernumerary elements that often occur in only 1 family among Carnivores, the Canidae [Pienkowska-Schelling et al., 2008; Trifonov et al., 2010; Dementyeva et al., 2012; http:// www.bionet.nsc.ru/labs/chromosomes/]. We also hope that in the framework of the Genome10K project other species with B chromosomes will be sequenced. These data will provide the groundwork for comparison of $\mathrm{B}$ chromosome composition in different orders and will shed light on B chromosome origins and the convergent and seemingly random nature of their occurrence in the mammalian tree.

Modern molecular cytogenetic techniques based on array and sequencing approaches, such as those recently developed for human, are also paving the way for comparative animal genetics. Soon these methods will be expanded for further study of genomes of many Carnivora species and will permit more efficient studies of chromosome evolution. The domestic dog SNP chip is now widely used, and a feline (F. catus) SNP chip just became available (www.morrisanimalfoundation.org).

Chromosome painting in Carnivora has laid down a solid foundation for describing major chromosome rearrangements and rates of chromosome exchanges, indicating signature associations for many taxa. Now we are coming to the exciting point in the comparative genomics story. In the near future whole-genome sequences for many Carnivora species will become available and hopefully chromosome assignment of scaffolds will not lag too far behind. This will provide rich resources to reveal the actual number of chromosomal rearrangements, especially intrachromosomal changes that occurred during evolution, that surpasses the resolution of chromosome painting and will provide insights into breakpoint region composition and into the origin and function of heterochromatin and B chromosomes.

\section{Acknowledgements}

This study was funded in part by programs MCB, RAS and SB RAS Programs and by research grants of Russian Fund for Basic Research. The authors thank 2 anonymous reviewers for helpful comments.

\section{References}

-Alekseyev MA, Pevzner PA: Breakpoint graphs and ancestral genome reconstructions. Genome Res 19:943-957 (2009).

Arnason U: The relationship between the four principal pinniped karyotypes. Hereditas 87 : 227-242 (1977).

-Arnason U, Adegoke JA, Gullberg A, Harley EH, Janke A, Kullberg M: Mitogenomic relationships of placental mammals and molecular estimates of their divergences. Gene 421:3751 (2008).

Avise JC, Robinson TJ: Hemiplasy: a new term in the lexicon of phylogenetics. Syst Biol 57: 503-507 (2008).

- Bardeleben C, Moore RL, Wayne RK: A molecular phylogeny of the Canidae based on six nuclear loci. Mol Phylogenet Evol 37:815-831 (2005).

-Biltueva LS, Yang F, Vorobieva NV, Graphodatsky AS: Comparative map between the domestic pig and dog. Mamm Genome 15: 809-818 (2004).

Breen M: Canine cytogenetics - from band to basepair. Cytogenet Genome Res 120:50-60 (2008).

Comparative Chromosome Painting in

Carnivora and Pholidota
Breen M, Thomas R, Binns MM, Carter NP, Langford CF: Reciprocal chromosome painting reveals detailed regions of conserved synteny between the karyotypes of the domestic dog (Canis familiaris) and human. Genomics 61:145-155 (1999).

reen M, Jouquand S, Renier C, Mellersh CS, Hitte C, et al: Chromosome-specific singlelocus FISH probes allow anchorage of an 1800-marker integrated radiation-hybrid/ linkage map of the domestic dog genome to all chromosomes. Genome Res 11:1784-1795 (2001).

Buckley-Beason VA, Johnson WE, Nash WG, Stanyon R, Menninger JC, et al: Molecular evidence for species-level distinctions in clouded leopards. Curr Biol 16:2371-2376 (2006).

Camacho JP, Sharbel TF, Beukeboom LW: Bchromosome evolution. Philos Trans R Soc Lond B Biol Sci 355:163-178 (2000).

-Cavagna P, Menotti A, Stanyon R: Genomic homology of the domestic ferret with cats and humans. Mamm Genome 11:866-870 (2000).
Che J, Wang J, Su W, Ye J, Wang Y, et al: Construction, characterization and FISH mapping of a bacterial artificial chromosome library of Chinese pangolin (Manis pentadactyla). Cytogenet Genome Res 122:55-60 (2008).

Dementyeva P, Makunin A, Graphodatsky AS, Trifonov V: Genes on B chromosomes of vertebrates. Genet Mol Biol, in press (2012).

Duke Becker SE, Thomas R, Trifonov VA, Wayne RK, Graphodatsky AS, Breen M: Anchoring the dog to its relatives reveals new evolutionary breakpoints across 11 species of the Canidae and provides new clues for the role of B chromosomes. Chromosome Res 19:685708 (2011).

Dutrillaux B, Couturier J: The ancestral karyotype of Carnivora: comparison with that of platyrrhine monkeys. Cytogenet Cell Genet 35:200-208 (1983).

Eizirik E, Murphy WJ, Koepfli KP, Johnson WE, Dragoo JW, et al: Pattern and timing of diversification of the mammalian order Carnivora inferred from multiple nuclear gene sequences. Mol Phylogenet Evol 56:49-63 (2010).

Cytogenet Genome Res 2012;137:174-193 
Ferguson-Smith MA: Genetic analysis by chromosome sorting and painting: Phylogenetic and diagnostic applications. Eur J Hum Genet 5:253-265 (1997).

Froenicke L: Origins of primate chromosomes as delineated by Zoo-FISH and alignments of human and mouse draft genome sequences. Cytogenet Genome Res 108:122-138 (2005).

-Frönicke L, Muller-Navia J, Romanakis K, Scherthan H: Chromosomal homeologies between human, harbor seal (Phoca vitulina) and the putative ancestral carnivore karyotype revealed by Zoo-FISH. Chromosoma 106:108-113 (1997).

Gaubert P, Antunes A: Assessing the taxonomic status of the Palawan pangolin Manis culionensis (Pholidota) using discrete morphological characters. J Mammalogy 86:10681074 (2005).

-Gaubert P, Cordeiro-Estrela P: Phylogenetic systematics and tempo of evolution of the Viverrinae (Mammalia, Carnivora, Viverridae) within feliformians: implications for faunal exchanges between Asia and Africa. Mol Phylogenet Evol 41:266-278 (2006).

Gaudin TJ, Wible JR: The entotympanic of pangolins and the phylogeny of the Pholidota. J Mamm Evol 6:39-65 (1999).

Gaudin TJ, Emry RJ, Wible JR: The phylogeny of living and extinct pangolins (Mammalia, Pholidota) and associated taxa: a morphology based analysis. J Mammal Evol 16:235305 (2009).

Grafodatsky AS, Biltueva LS: Homology of Gstained chromosomes of mammals. Genetika 23:93-103 (1987).

Graphodatsky AS, Radjabli SI: Chromosomes of argicultural and laboratory mammals. Atlas. (AN USSR, SB, Institute of Cytology and Genetics, Novosibirsk 1988).

Graphodatsky A, Sharshov A, Ternovsky D: Comparative cytogenetics of Mustelidae. Zoologichesky Zhurnal 68:96-106 (1989).

-Graphodatsky AS, Yang F, O’Brien PC, Serdukova N, Milne BS, et al: A comparative chromosome map of the arctic fox, red fox and dog defined by chromosome painting and high resolution G-banding. Chromosome Res 8:253-263 (2000a).

-Graphodatsky AS, Yang F, Serdukova N, Perelman P, Zhdanova NS, Ferguson-Smith MA: Dog chromosome-specific paints reveal evolutionary inter- and intrachromosomal rearrangements in the American mink and human. Cytogenet Cell Genet 90:275-278 (2000b).

-Graphodatsky AS, Yang F, O’Brien PC, Perelman P, Milne BS, et al: Phylogenetic implications of the 38 putative ancestral chromosome segments for four canid species. Cytogenet Cell Genet 92:243-247 (2001).

-Graphodatsky AS, Yang F, Perelman PL, O’Brien PC, Serdukova NA, et al: Comparative molecular cytogenetic studies in the order Carnivora: mapping chromosomal rearrangements onto the phylogenetic tree. Cytogenet Genome Res 96:137-145 (2002).
Graphodatsky AS, Kukekova AV, Yudkin DV, Trifonov VA, Vorobieva NV, et al: The protooncogene C-KIT maps to canid B-chromosomes. Chromosome Res 13:113-122 (2005).

Graphodatsky AS, Perelman PL, Sokolovskaya NV, Beklemisheva VR, Serdukova NA, et al: Phylogenomics of the dog and fox family (Canidae, Carnivora) revealed by chromosome painting. Chromosome Res 16:129143 (2008).

Hameister H, Klett C, Bruch J, Dixkens C, Vogel W, Christensen K: Zoo-FISH analysis: the American mink (Mustela vison) closely resembles the cat karyotype. Chromosome Res 5:5-11 (1997).

Johnson WE, Eizirik E, Pecon-Slattery J, Murphy WJ, Antunes A, et al: The late miocene radiation of modern Felidae: a genetic assessment. Science 311:73-77 (2006).

Kemkemer C, Kohn M, Cooper DN, Froenicke L, Hogel J, et al: Gene synteny comparisons between different vertebrates provide new insights into breakage and fusion events during mammalian karyotype evolution. BMC Evol Biol 9:84 (2009).

Koepfli KP, Gompper ME, Eizirik E, Ho CC, Linden L, et al: Phylogeny of the Procyonidae (Mammalia: Carnivora): molecules, morphology and the great American interchange. Mol Phylogenet Evol 43:1076-1095 (2007).

Koepfli KP, Deere KA, Slater GJ, Begg C, Begg K, et al: Multigene phylogeny of the Mustelidae: resolving relationships, tempo and biogeographic history of a mammalian adaptive radiation. BMC Biol 6:10 (2008).

Larkin DM, Pape G, Donthu R, Auvil L, Welge $\mathrm{M}$, Lewin HA: Breakpoint regions and homologous synteny blocks in chromosomes have different evolutionary histories. Genome Res 19:770-777 (2009).

Lindblad-Toh K, Wade CM, Mikkelsen TS, Karlsson EK, Jaffe DB, et al: Genome sequence, comparative analysis and haplotype structure of the domestic dog. Nature 438: 803-819 (2005).

Murphy WJ, Eizirik E, O’Brien SJ, Madsen O, Scally M, et al: Resolution of the early placental mammal radiation using Bayesian phylogenetics. Science 294:2348-2351 (2001a).

Murphy WJ, Stanyon R, O’Brien SJ: Evolution of mammalian genome organization inferred from comparative gene mapping. Genome Biol 2:REVIEWS0005 (2001b).

Nash WG, Wienberg J, Ferguson-Smith MA, Menninger JC, O’Brien SJ: Comparative genomics: tracking chromosome evolution in the family Ursidae using reciprocal chromosome painting. Cytogenet Cell Genet 83: 182-192 (1998).

Nash WG, Menninger JC, Wienberg J, PadillaNash HM, O’Brien SJ: The pattern of phylogenomic evolution of the Canidae. Cytogenet Cell Genet 95:210-224 (2001).

-Nash WG, Menninger JC, Padilla-Nash HM, Stone G, Perelman PL, O’Brien SJ: The ancestral carnivore karyotype $(2 \mathrm{n}=38)$ lives today in ringtails. J Hered 99:241-253 (2008).
Nie W, Wang J, O’Brien PC, Fu B, Ying T, et al: The genome phylogeny of domestic cat, red panda and five mustelid species revealed by comparative chromosome painting and G-banding. Chromosome Res 10:209-222 (2002).

Nie W, Wang J, Perelman P, Graphodatsky AS, Yang F: Comparative chromosome painting defines the karyotypic relationships among the domestic dog, Chinese raccoon dog and Japanese raccoon dog. Chromosome Res 11: 735-740 (2003).

Nie W, Wang J, Su W, Wang Y, Yang F: Chromosomal rearrangements underlying karyotype differences between Chinese pangolin (Manis pentadactyla) and Malayan pangolin (Manis javanica) revealed by chromosome painting. Chromosome Res 17:321-329 (2009).

- Nie W, Wang J, Su W, Wang D, Tanomtong A, et al: Chromosomal rearrangements and karyotype evolution in carnivores revealed by chromosome painting. Heredity 108:1727 (2012).

Perelman PL, Graphodatsky AS, Serdukova NA, Nie W, Alkalaeva EZ, et al: Karyotypic conservatism in the suborder Feliformia (order Carnivora). Cytogenet Genome Res 108: 348-354 (2005).

- Perelman PL, Graphodatsky AS, Dragoo JW, Serdyukova NA, Stone G, et al: Chromosome painting shows that skunks (Mephitidae, Carnivora) have highly rearranged karyotypes. Chromosome Res 16:1215-1231 (2008).

-Pienkowska-Schelling A, Schelling C, Zawada M, Yang F, Bugno M, Ferguson-Smith M: Cytogenetic studies and karyotype nomenclature of three wild canid species: maned wolf (Chrysocyon brachyurus), bat-eared fox (Otocyon megalotis) and fennec fox (Fennecus zerda). Cytogenet Genome Res 121:25-34 (2008).

Qin XM, Dou SR, Guan QX, Qin PS, She Y: Complete mitochondrial genome of the Manis pentadactyla (Pholidota, Manidae): comparison of M. pentadactyla and M. tetradactyla. Mitochondrial DNA 23:37-38 (2012).

Rettenberger G, Klett C, Zechner U, Bruch J, Just W, et al: Zoo-FISH analysis: Cat and human karyotypes closely resemble the putative ancestral mammalian karyotype. Chromosome Res 3:479-486 (1995).

Robinson TJ, Ruiz-Herrera A, Avise JC: Hemiplasy and homoplasy in the karyotypic phylogenies of mammals. Proc Natl Acad Sci USA 105:14477-14481 (2008).

Rocchi M, Archidiacono N, Schempp W, Capozzi O, Stanyon R: Centromere repositioning in mammals. Heredity 108:59-67 (2012).

Sargan DR, Yang F, Squire M, Milne BS, O’Brien PC, Ferguson-Smith MA: Use of flow-sorted canine chromosomes in the assignment of canine linkage, radiation hybrid, and syntenic groups to chromosomes: refinement and verification of the comparative chromosome map for dog and human. Genomics 69: 182-195 (2000). 
Sillero-Zubiri C, Hoffmann M, Macdonald DW: Canids: Foxes, Wolves, Jackals and Dogs. Status Survey and Conservation Action Plan. 2004. (IUCN/SSC Canid Specialist Group, Gland, Switzerland and Cambridge, UK 2004).

Stanyon R: Potos flavus, in O'Brien SJ, Menninger JC, Nash WG (eds): Atlas of Mammalian Chromosomes, p 482 (John Wiley and Sons, New York 2006).

-Switonski M, Szczerbal I, Nowacka-Woszuk J: Comparative genomics of 3 farm canids in relation to the dog. Cytogenet Genome Res 126:86-96 (2009).

-Szczerbal I, Kaczmarek M, Switonski M: Compound mosaicism, caused by B chromosome variability, in the Chinese raccoon $\operatorname{dog}(\mathrm{Nyc}$ tereutes procyonoides procyonoides). Folia Biol (Krakow) 53:155-159 (2005).

-Thomas R, Breen M, Langford CF, Binns MM: Zoo-FISH analysis of dog chromosome 5:identification of conserved synteny with human and cat chromosomes. Cytogenet Cell Genet 87:4-10 (1999).

- Tian Y, Nie WH, Wang JH, Yang YF, Yang FT: Comparative chromosome painting shows the red panda (Ailurus fulgens) has a highly conserved karyotype. Yi Chuan Xue Bao 29: 124-127 (2002).

-Tian Y, Nie W, Wang J, Ferguson-Smith MA, Yang F: Chromosome evolution in bears: reconstructing phylogenetic relationships by cross-species chromosome painting. Chromosome Res 12:55-63 (2004).
Trifonov VA, Perelman PL, Kawada SI, Iwasa MA, Oda SI, Graphodatsky AS: Complex structure of B-chromosomes in two mammalian species: Apodemus peninsulae (Rodentia) and Nyctereutes procyonoides (Carnivora). Chromosome Res 10:109-116 (2002).

Trifonov VA, Dementyeva PV, Beklemisheva VR, Yudkin DV, Vorobieva NV, Graphodatsky AS: Supernumerary chromosomes, segmental duplications, and evolution. Russ J Genet 46:1094-1096 (2010).

Vujosevic M, Blagojevic J: B chromosomes in populations of mammals. Cytogenet Genome Res 106:247-256 (2004).

Wayne RK, Nash WG, O’Brien SJ: Chromosomal evolution of the Canidae. I. Species with high diploid numbers. Cytogenet Cell Genet 44: 123-133 (1987).

Wienberg J, Stanyon R, Nash WG, O'Brien PC, Yang F, et al: Conservation of human vs. feline genome organization revealed by reciprocal chromosome painting. Cytogenet Cell Genet 77:211-217 (1997).

Wilson DE, Reeder DM: Mammal Species of the World. A Taxonomic and Geographic Reference, ed 3 (Johns Hopkins University Press, Baltimore 2005).

-Wurster-Hill DH: Chromosomes of eight species from five families of Carnivora. J Mammal 54:753-760 (1973).

Wurster-Hill DH, Centerwall WR: The interrelationships of chromosome banding patterns in canids, mustelids, hyena, and felids. Cytogenet Cell Genet 34:178-192 (1982).

-Wurster-Hill DH, Gray CW: Giemsa banding patterns in the chromosomes of twelve species of cats (Felidae). Cytogenet Cell Genet 12:388-397 (1973).
Wurster-Hill DH, Gray CW: The interrelationships of chromosome banding patterns in procyonids, viverrids, and felids. Cytogenet Cell Genet 15:306-331 (1975).

Yang F, Graphodatsky AS: Integrated comparative genome maps and their implications for karyotype evolution of carnivores, in Schmid M, Nanda I (eds): Chromosomes Today, vol 14, pp 215-224 (Kluwer Academic Publishers, Dordrecht 2004).

-Yang F, O’Brien PC, Milne BS, Graphodatsky AS, Solanky N, et al: A complete comparative chromosome map for the dog, red fox, and human and its integration with canine genetic maps. Genomics 62:189-202 (1999).

-Yang F, Graphodatsky AS, O’Brien PC, Colabella A, Solanky N, et al: Reciprocal chromosome painting illuminates the history of genome evolution of the domestic cat, dog and human. Chromosome Res 8:393-404 (2000).

- Yang F, Graphodatsky AS, Li T, Fu B, Dobigny G, et al: Comparative genome maps of the pangolin, hedgehog, sloth, anteater and human revealed by cross-species chromosome painting: Further insight into the ancestral karyotype and genome evolution of eutherian mammals. Chromosome Res 14:283296 (2006).

-Yudkin DV, Trifonov VA, Kukekova AV, Vorobieva NV, Rubtsova NV, et al: Mapping of KIT adjacent sequences on canid autosomes and $\mathrm{B}$ chromosomes. Cytogenet Genome Res 116:100-103 (2007).

Zrzavy J, Ricankova V: Phylogeny of recent Canidae (Mammalia, Carnivora): relative reliability and utility of morphological and molecular datasets. Zool Scr 33:311-333 (2004). 